\title{
Magnetic metal-complex-conducting copolymer core-shell nanoassemblies for a single-drug anticancer platform
}

\author{
Jeong-Hwan Kim ${ }^{1}$, Haruki Eguchi ${ }^{2}$, Masanari Umemura $^{1}$, Itaru Sato ${ }^{1,3}$, Shigeki Yamada ${ }^{4}$, Yujiro Hoshino ${ }^{5}$, \\ Takatsugu Masuda $^{6}$, Ichio Aoki ${ }^{7}$, Kazuo Sakurai ${ }^{8}$, Masahiro Yamamoto ${ }^{9}$ and Yoshihiro Ishikawa ${ }^{1}$
}

Nanoparticulate agents for magnetic drug delivery systems (DDSs) have extensive applications in targeted drug delivery, contrast imaging and therapeutics. However, no simple synthetic method for magnetic DDS agents has been developed without the need to add magnetic nanoparticles. Here, we describe the one-step fabrication of 'all-in-one' magneto-assemblies using an 'inorganic-metal-salt-free' method, involving spontaneous self-assembly of the water-insoluble prodrug $\mu$-oxo-bis( $N, N$-ethylenebis (salicylideniminato)iron) [Fe(salen)] (magnetic core) with polypyrrole (PPy)-b-polycaprolactone (PCL) smart diblock copolymers. In the system, PCL serves as a heat-responsive core scaffold, and PPy serves as an electronic core-size controller and $\mathrm{pH}$-responsive shell. This core-shell nanocomposite has a high-loading capacity $(\sim 90 \%)$, and the core size is tunable by incorporating albumin or gum arabic as bio-coating agents, which also provide colloidal stability, biocompatibility and thermo-stability. $\mathrm{Fe}$ (salen), which has intrinsic antitumor activity, also has ubiquitous magnetic properties, which are dramatically enhanced in these molecular assemblies with magnetic coupling. Moreover, these multifunctional nanoassemblies can be delivered magnetically, can serve as magnetic resonance imaging contrast agents, can generate magneto-hyperthermal effects and can enable magnetic field-triggered release of $\mathrm{Fe}($ salen) molecules under acidic conditions.

NPG Asia Materials (2017) 9, e367; doi:10.1038/am.2017.29; published online 31 March 2017

\section{INTRODUCTION}

Local drug delivery systems (DDSs) using magnetic nanoarchitectures are at the leading edge of materials science, nanotechnology and nanomedicine, and provide improved diagnostic and therapeutic efficacy while minimizing side effects. ${ }^{1-6}$ Recent advances in block copolymer-based self-assembly $^{7-9}$ have led to the development of various magnetic DDS vehicles ${ }^{1-6}$ such as polymeric nanocarriers into which prodrugs are often co-encapsulated with magnetic nanoparticles (MNPs), and these nanocarriers serve to shield the drug and to enable its magnetically guided imaging and local release in response to an alternating magnetic field (AMF).

However, the synthesis of MNPs with desirable magnetic properties for magnetic DDS platforms requires numerous steps to control the particles' core, diameter, anisotropy, suspension composition and surface coating, ${ }^{1-9}$ and requires considerable care to avoid adverse effects in vivo. For example, an excessive dose of MNPs can cause oxidative stress-induced cell injury, ${ }^{10,11}$ and slow degradation can lead to accumulation in the liver and other organs, ${ }^{5,12}$ resulting in potentially fatal effects such as cardiovascular damage, blood clots and hypersensitivity. Undoubtedly, one of the major hurdles is that MNPs are usually large, causing co-loading of hydrophobic drugs to be restricted and encapsulation efficiency to be low. Even the nanocarrier shows limited morphological tunability and poor drug loading $(<20 \%)$ due to the high proportion of carrier materials required for drug loading. ${ }^{5,6}$ To address these problems, a single low-molecular-weight drug component having multifunctional capabilities is required. Metal complexes are useful for this purpose $^{13-16}$ because a metal complex is not only small but also can be therapeutically active with magnetic ${ }^{13,14}$ or optical properties. ${ }^{15,16}$ Therefore, a single-step fabrication method for a metal-salt-free, magnetic metal-complex-based local DDS platform might shift the synthetic paradigm to realize advanced DDS applications.

We have previously reported a metal-complex-based DDS agent, $\mathrm{Fe}$ (salen) nanoparticles in a water suspension, ${ }^{13,14}$ which proved suitable for magnetic DDS, chemotherapy, magnetic resonance imaging (MRI) and AMF-induced local hyperthermal therapy.

\footnotetext{
${ }^{1}$ Cardiovascular Research Institute, Yokohama City University, Graduate School of Medicine, Yokohama, Japan; ${ }^{2}$ Department of Advanced Applied Science, Research Laboratory, IHI Corporation, Yokohama, Japan; ${ }^{3}$ Department of Oral Surgery, Yokohama City University, Graduate School of Medicine, Yokohama, Japan; ${ }^{4}$ Department of Materials System Science, International Graduate School of Arts and Science, Yokohama City University, Yokohama, Japan; ${ }^{5}$ Department of Environment and Natural Sciences, Graduate School of Environment and Information Sciences, Yokohama National University, Yokohama, Japan; ${ }^{6}$ Tokyo Institute for Solid State Physics, the University of Tokyo, Tokai, Japan; ${ }^{7}$ Department of Molecular Imaging and Theranostics, National Institute of Radiological Sciences, Chiba, Japan; ${ }^{8}$ Department of Chemistry and Biochemistry, The University of Kitakyushu, Fukuoka, Japan and ${ }^{9}$ Faculty of Science and Engineering, Department of Chemistry of Functional Molecules, Konan University, Kobe, Japan

Correspondence: Professor J-H Kim or Y Ishikawa, Cardiovascular Research Institute, Yokohama City University, Graduate School of Medicine, 3-9 Fukuura, Kanazawa-ku, Yokohama 236-0004, Japan.

E-mail: jeongkim@yokohama-cu.ac.jp or yishikaw@med.yokohama-cu.ac.jp

Received 24 August 2016; revised 21 November 2016; accepted 28 December 2016
} 


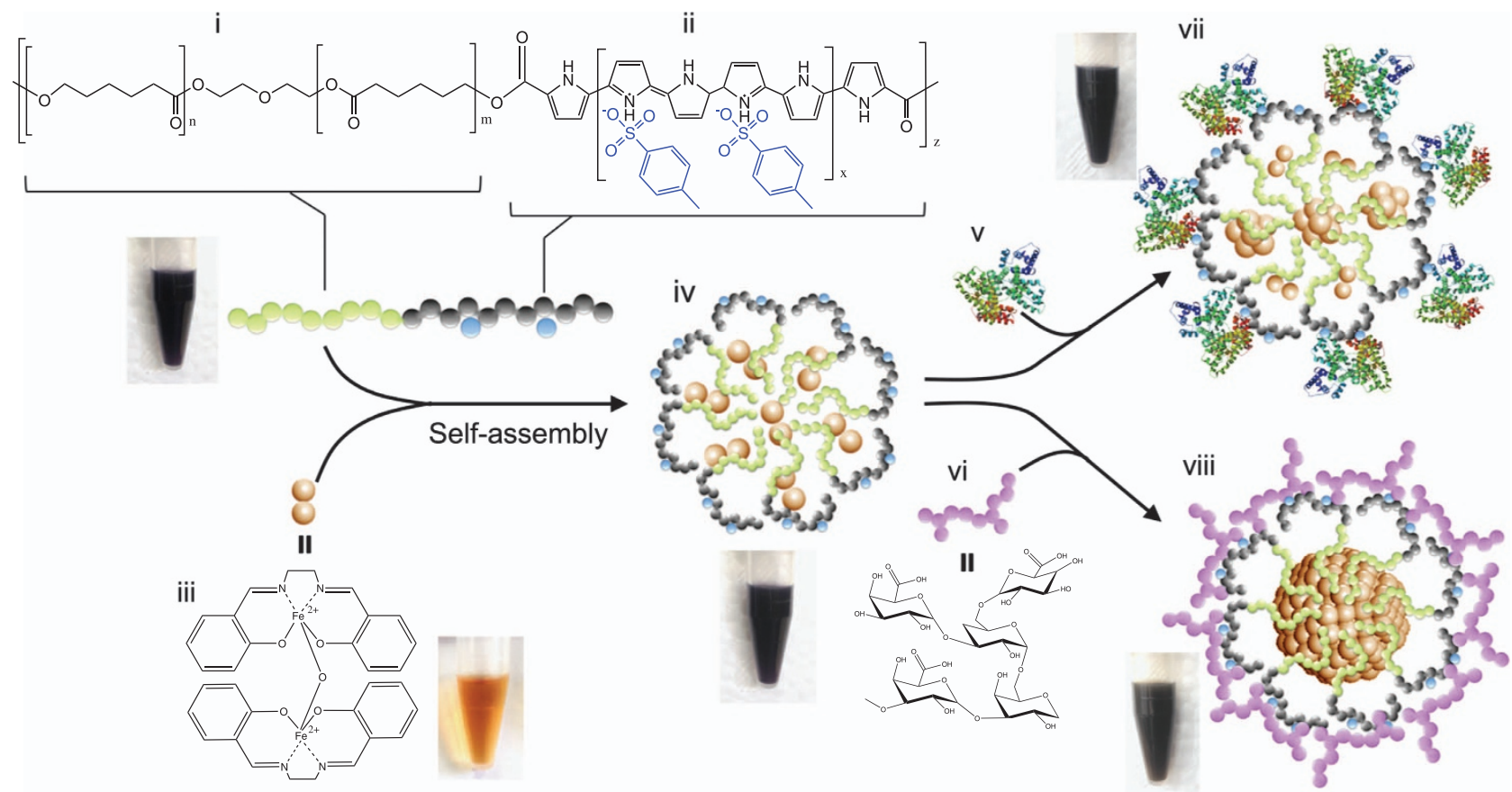

Scheme 1 Preparation of Fe(salen)-loaded polypyrrole (PPy)-polycaprolactone (PCL) core-shell nanoassembled composites (iv) and the modulation of the core-size induced by bio-functionalization with bovine serum albumin (BSA) (vii) and gum arabic (GA) (viii). (i) PCL; (ii) PPy (benzosulfonate-doped); (iii) Fe(salen); (iv) Fe(salen)-loaded nanoassemblies; (v) BSA; (vi) GA; (vii) BSA-coated Fe(salen)-loaded nanoassemblies; (viii) GA-coated Fe(salen)-loaded nanoassemblies. Photos of suspension are displayed at each step of formulation.

Compared with traditional MNP-based methods that require additional co-loading processes of drug reagents, ${ }^{1-9}$ the $\mathrm{Fe}$ (salen)-based system is unprecedented in that a single agent provides both the magnetic properties and anticancer activity. In other words, the $\mathrm{Fe}$ (salen) metal complexes, which have been used in the last few decades as asymmetric catalysts ${ }^{17-20}$ and molecular magnets, ${ }^{21,22}$ can be employed as magnetically guided anticancer agents. ${ }^{13,14}$ Other molecular magnets have been reported but cannot be practically applied due to the very low critical temperature at which they exhibit positive values of magnetization in response to a room temperature magnetic field. ${ }^{23-26}$ However, Fe(salen) also has some drawbacks, such as poor water solubility, loss of magnetic properties in solvents and potential cytotoxicity when accumulated in organs. Herein, we report a simple, 'inorganic-metal-salt-free', one-step synthesis of a magnetic core-shell nanoassembly (hereafter CSNA) DDS nanocarrier, comprising $\mathrm{Fe}$ (salen) as a prodrug core, a biodegradable polypyrrole (PPy)-polycaprolactone (PCL)-shell (referred to as PP), and bovine serum albumin (BSA) or gum arabic (GA; Scheme 1).

The PP-copolymer immediately self-assembles to encapsulate the $\mathrm{Fe}$ (salen) molecules at $25^{\circ} \mathrm{C}$, and the morphology of the prodrug cores can be readily controlled by bio-functionalization. The orange color of the $\mathrm{Fe}$ (salen) solution immediately changes to black, which is a useful optical indicator of micelle-like nanoassembly. When $\mathrm{Fe}$ (salen)-dispersion is mixed with PP in a methanol solution, the PP-copolymer self-assembles to encapsulate $\mathrm{Fe}$ (salen) in the core, which did not require heating or the use of toxic solvents. Further, in the presence of BSA or GA, small nanoclusters (NCs; vii) or large NCs (viii), respectively, are formed in a controlled fashion. This nanocarrier functions as a single-drug multifunctional DDS vehicle for magnetically guided delivery, MRI, magneto-hyperthermia and triggered release of anticancer-active $\mathrm{Fe}$ (salen) molecules.

\section{MATERIALS AND METHODS}

\section{Reagents and methods}

$\mathrm{Fe}$ (salen) was purchased from Tokyo Chemical Industry (TCI) Co., Ltd, Japan. Other chemicals including the biodegradable PPy- $b$-PCL copolymer (PCL diol blocks and PPy blocks have average molecular weights of 2000 and 4000, respectively; PPy domains were $25 \%$ doped with $p$-toluenesulfonate) were purchased from Sigma-Aldrich (Saint Louis, MO, USA). Sample preparation was conducted under ambient conditions (room temperature).

\section{General procedure for the preparation of $\mathrm{Fe}$ (salen)-load CSNAs}

$\mathrm{Fe}$ (salen) powder $(10 \mathrm{mg}$ ) was dissolved in $100 \mathrm{ml}$ of methanol. The solution was stirred thoroughly and then centrifuged at 150000 r.p.m., followed by a neodymium magnetic separation to discard any magnetite-like sediments. The clear solution was filtered to remove any undissolved material. PPy- $b$-PCL ( $2.5 \mathrm{ml}$ of $0.5 \mathrm{wt} \%$, Sigma-Aldrich) was briefly sonicated with a hornultrasonicator for $10 \mathrm{~s}$ (pulsed mode) and added to the $\mathrm{Fe}($ salen) solution. After vigorous stirring for $15 \mathrm{~min}$, black PPy- $b$-PCL-Fe(salen) was precipitated and collected by a neodymium magnetic separation to ensure it was free of unloaded $\mathrm{Fe}$ (salen) or PPy- $b$-PCL ( $\mathrm{Fe}$ (salen) dissolved in methanol is not attracted by a magnet due to its anti-ferromagnetism). When Fe(salen) was dispersed in aqueous solution (saline), it immediately aggregated due to its hydrophobic nature, exhibiting ferromagnetic-like behavior by the attachment of a magnet, in accordance with our previous reports. ${ }^{13,14}$ Prolonged exposure to air ( $>7$ days) during the recrystallization process caused a significant loss of magnetism of $\mathrm{Fe}$ (salen) NPs, presumably due to oxidation. On the other hand, the CSNAs showed stable magnetism for at least 6 months of subsequent air exposure, implying that the formation of the homogeneous PPy-shell prevents oxidative modification of the $\mathrm{Fe}$ (salen) core.

\section{Preparation of BSA- or GA-coated Fe(salen)-loaded CSNAs}

The black precipitate was washed several times with methanol and redispersed in $50 \mathrm{ml}$ of saline with sonication for $\sim 10 \mathrm{~s}$ (pulsed mode). An excess amount of dispersant, either BSA (Sigma-Aldrich, $50 \mathrm{mg}$, MW $66.5 \mathrm{kDa}$ ) or GA (Sigma-Aldrich, $50 \mathrm{mg}$, MW $250 \mathrm{kDa}$ ), was then added. The reaction mixture was sonicated for $10 \mathrm{~s}$ in pulsed mode, ultracentrifuged at 
150000 r.p.m. for $30 \mathrm{~min}$, and washed with the aid of magnetic separation three times to ensure removal of any uncoated dispersant molecules.

$\mathrm{X}$-ray diffraction and small-angle $\mathrm{X}$-ray scattering characterization The X-ray diffraction (XRD) and small-angle X-ray scattering measurements were performed with a Rigaku horizontal XRD apparatus SmartLab with a $\mathrm{Cu}$ target (output: $45 \mathrm{kV}-200 \mathrm{~mA}$ ). Iron-oxide particles $\left(\mathrm{Fe}_{3} \mathrm{O}_{4} / \mathrm{Fe}_{2} \mathrm{O}_{3}\right.$, Sigma-Aldrich) were used as a standard for XRD.

\section{X-ray photoelectron spectroscopy}

$\mathrm{X}$-ray photoelectron spectroscopy (XPS) analysis was carried out using an ESCA5500MC spectrometer (ULVAC-PHI, Inc., Kanagawa, Japan) to examine the valence state, that is, oxidation state, of $\mathrm{Fe}($ salen).

\section{HRTEM analysis}

The particle size and structure of the crystalline $\mathrm{Fe}($ salen) sample were observed using a Gatan Titan high-resolution transmission electron microscope (HRTEM, FEI, Pleasanton, CA, US) operating at 100-300 kV. The crystallographic orientations were measured by selected area electron diffraction (SAED) and fast Fourier transform (FFT) at an acceleration voltage of $200 \mathrm{kV}$ and a probe spot of several hundred micrometers in diameter. Samples for SAED and FFT observations were prepared by applying purified Fe(salen) powder on a film of amorphous carbon. Unit cell parameters were determined by SAED and with an X-ray diffractometer (RAD-IIB, Rigaku). To increase the HRTEM signal-to-noise ratio and improve crystal contrast, Fourier filtering of the raw HRTEM images was employed. All HRTEM images were taken on a slow-scan CCD camera $(14 \times 14 \mu \mathrm{m}, 2048 \times 2048$ pixels, Gatan UltraScan1000). A Tridem Gatan electron energy loss spectroscopy system with $0.7 \mathrm{eV}$ energy resolutions was used for $\mathrm{Fe}, \mathrm{N}, \mathrm{O}$ and $\mathrm{C}$ trace monitoring on a $\mathrm{Si}_{3} \mathrm{~N}_{4}$ TEM grid substrate (Ted Pella Inc., Redding, CA, USA).

\section{Magnetic characterization}

Magnetization versus magnetic field curves were obtained using a superconducting quantum interference device (Quantum Design MPMS7 system, San Diego, CA, USA). Each sample was enclosed in a plastic capsule (Quantum Design) for measurement. The curves for $\mathrm{Fe}$ (salen) and the CSNAs were plotted from 2 to $310 \mathrm{~K}$. Changes in magnetization $(\mathrm{M}(\mu / \mathrm{Fe}$ site) or $\mathrm{M}(\mathrm{emu} /$ g)) with applied magnetic field (H/Oe) were examined in the range of -10000 to 10000 Oe. For detecting energy absorption and magnetic intensity of the CSNA samples, electron spin resonance (ESR) analysis was performed using a BioSpin ESR EMX-8/2.7 system (Bruker, Billerica, MA, USA), operating at a $9.9 \mathrm{GHz}$ modulation frequency at room temperature. The microwave power and modulation amplitude were $1 \mathrm{~mW}$ and $1 \mathrm{G}$, respectively. Powder samples were prepared in a glass capillary sample holder and placed in a probe head (four measurements per sample). For the zero field cooling (ZFC) analysis, first the temperature was lowered to $2 \mathrm{~K}$ and a magnetic field was applied. Then, the magnetization was measured as the sample was heated to $300 \mathrm{~K}$ (ZFC). For the field cooling analysis, after heating to $300 \mathrm{~K}$ and applying a magnetic field, magnetization was measured as the temperature was lowered to $2 \mathrm{~K}$ (field cooling).

\section{MRI and magneto-relaxivity}

MRI measurements were performed in vitro. BSA- and GA-CSNAs, diluted with $10 \mathrm{~mm}$ phosphate-buffered saline, were prepared and aliquoted into 0.2-ml PCR tubes. MRI acquisition was performed on a 7.0-Tesla, 40-cm bore magnet (Kobelco and Jastec, Kobe, Japan) interfaced to an Avance-I system (Bruker-Biospin, Ettlingen, Germany) with a 35-mm coil (Rapid Biomedical, Lymper, Germany). The sample temperature was maintained at $23^{\circ} \mathrm{C}$ using a gradient-coil cooling system and air conditioner. For the $\mathrm{T}_{2}$-weighted images (T2W) and $\mathrm{T}_{2}$ calculations, two-dimensional, single-slice images were obtained using a multi-echo spin-echo sequence with the following parameters: repetition time $(\mathrm{TR})=3000 \mathrm{~ms}$, echo time $(\mathrm{TE})=10-100 \mathrm{~ms}$ in steps of $10 \mathrm{~ms}$ ( 10 echoes), matrix size $=256 \times 128$, field of view $=38.4 \times 19.2 \mathrm{~mm}^{2}$, slice thickness $=2.0 \mathrm{~mm}$, number of acquisitions $(\mathrm{NA})=1$ and slice orientation $=$ horizontal. For this imaging sequence, the nominal voxel resolution was
$150 \times 150 \times 2000 \mu^{3}$. The total acquisition time for $\mathrm{T} 2 \mathrm{~W}$ was $6 \mathrm{~min}$ and $24 \mathrm{~s}$. After image acquisition, the values of $T_{2}$ and $R_{2}$ were estimated using the MRVision image processing software (version 1.6.8, MR vision Co., MA, USA). The transverse relaxivity $\left(r_{2}\right)$ was calculated with the equation $r_{2}=\left(\mathrm{R}_{2 \mathrm{obs}}-\mathrm{R}_{2 \mathrm{~d}}\right) /[\mathrm{Fe}] \quad\left(\mathrm{R}_{2 \mathrm{obs}}=\mathrm{R}_{2}\right.$ of the sample, $\mathrm{R}_{2 \mathrm{~d}}=\mathrm{R}_{2}$ of the aqueous solution, $[\mathrm{Fe}]=\mathrm{Fe}$ concentration measured by ICP-MS). For the calculation of $\mathrm{T}_{1}$, two-dimensional single-slice inversion recovery MRI was performed using rapid acquisition with relaxation enhancement with the following parameters: $\mathrm{TR}=10000 \mathrm{~ms}$; effective- $\mathrm{TE}=20 \mathrm{~ms}$; inversion time $=52,100$, $200,400,800,1600,3200$ or $6400 \mathrm{~ms}$; matrix size $=128 \times 64$; slice orientation $=$ coronal; field of view $=38.4 \times 19.2 \mathrm{~mm}^{2}$; slice thickness $=2.0 \mathrm{~mm}$; rapid acquisition with relaxation enhancement factor $=4$; and $N A=1$. For this imaging, the nominal voxel resolution was $300 \times 300 \times 2000 \mu \mathrm{m}^{3}$. The total acquisition time for the $\mathrm{T}_{1}$ calculation was $21 \mathrm{~min}$ and $20 \mathrm{~s}$. Quantitative $\mathrm{T}_{1}$ maps were calculated by performing nonlinear least-squares fitting using inversion recovery MRI. The $\mathrm{R}_{1}$ values in the ROI were calculated as the inverse of the $\mathrm{T}_{1}$ values. All calculations and analyses were performed using the MRVision image analysis software (Version 1.5.8, MRVision Co., MA, USA).

\section{Inductively coupled plasma mass spectrometry (ICP-MS)}

ICP-MS (Agilent 4500, Agilent Technologies, Santa Clara, CA, USA) was used to determine the $\mathrm{Fe}($ salen) atomic-mass $\%$ of the $\mathrm{Fe}($ salen)-loaded CSNAs.

\section{Optical properties of $\mathrm{Fe}$ (salen)-CSNAs}

The ultraviolet-visible (UV-vis) absorbance and fourier transform infrared (FT-IR) characteristics of the CSNAs were determined using a Nanodrop UV-vis spectrophotometer (Fisher-Scientific, Waltham, MA, USA) and an FTIR spectrophotometer (JASCO FT/IR-4100) in ATR mode, respectively.

Colloidal size distribution and stability of Fe(salen)-CSNA samples Dynamic light scattering measurements were performed with a Malvern Zetasizer Nano ZS (Malvern, Herrenberg, Germany) equipped with a $633-\mathrm{nm}$ He-Ne laser operating at an angle of $173^{\circ}$. Five hundred microliters of each sample was measured in single-use polystyrene half-micro cuvettes (Thermon-Fisher Landsmeer, The Netherlands) with a path length of $10 \mathrm{~mm}$. The measurements were taken $4.65 \mathrm{~mm}$ from the cuvette wall with an automatic attenuator and at a controlled temperature of $25^{\circ} \mathrm{C}$. For each sample, 15 runs of $10 \mathrm{~s}$ were performed, with three repetitions for all CSNAs.

\section{Cell culture and proliferation assay}

Human synovial sarcoma (HS-SY-II) and human ovarian endometrioid carcinoma (OVK18) cell lines were purchased from the Health Science Research Resources Bank (Japan Health Sciences Foundation, Tokyo, Japan). The human glioblastoma (U87) cell line was obtained from the American Type Culture Collection (Virginia, USA). In all cases, early passage cultures were stored and used for experiments. These cell lines $\left(6 \times 10^{4}\right.$ or $3 \times 10^{5}$ cells) were seeded in RPMI-1640 with L-glutamine and phenol red medium containing $10 \%$ fetal bovine serum and $1 \%$ penicillin-streptomycin. The cell viability assay was performed using a commercial kit, the sodium 2,3-bis(2-methoxy-4-nitro5-sulfophenyl)-5-[(phenylamino)-carbonyl]-2H-tetrazolium inner salt (XTT) assay cell proliferation kit (American Type Culture Collection). The cytotoxic effect of AMF-induced hyperthermia was monitored with a pre-clinical in vivo imaging system.

\section{Alternating magnetic field-induced in vitro hyperthermia}

For the measurement of magnetically induced hyperthermia, AMF was generated by a vertical coil with an inner diameter of $4 \mathrm{~cm}$, driven by a transistor inverter (Hot Shot, Ameritherm Inc., Scottsville, NY, USA). Experiments were performed at a frequency of $307-308 \mathrm{kHz}$ and $378.8 \mathrm{~A}$, unless otherwise specified. ${ }^{14} \mathrm{~A}$ thermometer (hand-held thermometer HA-200, Anritsu Meter Co., Tokyo, Japan) or a thermograph (InfraRed camera, Nippon Avionics Co., Ltd., Tokyo, Japan) was used to determine temperature in vitro. ${ }^{14}$ For the in vivo imaging system test, $2.5 \mathrm{mg} \mathrm{ml}^{-1}$ of the BSA-CSNA sample was prepared with saline solution, and $1 \mathrm{ml}$ of the sample solution was added into a cell culture dish ( $\sim 5000$ cells) containing $3 \mathrm{ml}$ of DMEM buffer 
(final concentration of $0.625 \mathrm{mg} \mathrm{ml}^{-1}$ ). For the $\mathrm{pH}$-dependent AMF-hyperthermal platform, the U87 cell culture media were adjusted to either $\mathrm{pH} 7.4$ or $\mathrm{pH} 6.0$ immediately before introducing the BSA-CSNA samples. The BSA-CSNA samples, at concentrations of $625 \mu \mathrm{g} \mathrm{ml}^{-1}$, were briefly sonicated and introduced into $1 \mathrm{ml}$ of cancer cell culture media $\left(3 \times 10^{5}\right.$ cells per $4 \mathrm{~cm}$ pellet). The cells without BSA-CSNAs and AMF-treatment were used as a control. The cells were subjected to an AMF-hyperthermia treatment for 30 or $60 \mathrm{~min}$ to verify the individual/ combined effect of the BSA-CSNAs on the cell viability. The frequency and current were optimized at $280 \mathrm{kHz}$ and $335.4 \mathrm{~A}$, respectively. Following the AMF-treatment, the cells were grown for $24 \mathrm{~h}$. The viability of the cells was evaluated by counting live/dead cells with a TC20 automated cell counter (BioRad, Hercules, CA, USA). Following cell staining according to the manufacturer's protocol (Cell Counting Kit-8, Dojindo, Tokyo, Japan), bright field/fluorescence imaging was carried out using an inverted Nikon Eclipse Ti confocal microscope (Melville, NY, USA).

\section{Quantitation of loading/release of Fe(salen)}

The drug-loading capacity (DLC) (\%) was measured as $\left(\mathrm{M}_{\text {initialFe(salen) }}{ }^{-}\right.$ $\left.\mathrm{M}_{\mathrm{Fe}(\text { salen }) \text { in excess }}\right) \times 100 /\left(\mathrm{M}_{\mathrm{CSNA}}\right)\left(\mathrm{mg} \mathrm{g}^{-1}\right)$, where $\mathrm{M}_{\text {initialFe(salen) }}$ is the initial mass of the added $\mathrm{Fe}$ (salen), $\mathrm{M}_{\mathrm{Fe}(\text { salen) in excess }}$ is the mass of the $\mathrm{Fe}($ salen) in the supernatant and $\mathrm{M}_{\mathrm{CSNA}}$ is the mass of the $\mathrm{Fe}$ (salen)-loaded CSNA samples. The drug-loading efficiency (\%) was measured as $\left(\mathrm{M}_{\text {initialFe(salen) }}-\mathrm{M}_{\mathrm{Fe}(\mathrm{salen}) \text { in }}\right.$ excess $) \times 100 /\left(\mathrm{M}_{\text {initialFe(salen })}\right)\left(\mathrm{mgg}^{-1}\right)$. The release activity of the $\mathrm{Fe}($ salen $)$ loaded CSNAs was monitored by measuring the time course of the UV-vis absorbance at $\lambda_{\max }=310 \mathrm{~nm}$ at various temperatures. At each pre-determined interval, the sample was centrifuged, and its UV/vis absorbance at $\lambda_{\max }=310 \mathrm{~nm}$ was used to quantify the amount of $\mathrm{Fe}($ salen) released from the supernatant, according to a pre-determined calibration curve. All of the release profiles are shown in cumulative mode.

\section{RESULTS AND DISCUSSIONS}

\section{Structural analyses of crystalline Fe(salen)}

To establish the purity of $\mathrm{Fe}($ salen), crystals obtained from acetone were characterized by XRD analysis, as shown in Figure 1a. No characteristic signals of magnetite were seen, based on the standard reflections for $\mathrm{Fe}_{3} \mathrm{O}_{4} / \mathrm{Fe}_{2} \mathrm{O}_{3}$. The recrystallized $\mathrm{Fe}$ (salen) has a triclinic crystal structure $\left(\alpha, \beta\right.$ and $\left.\gamma \neq 90^{\circ}\right)$ due to $\mathrm{Fe}$ (salen) dimer formation, as reported earlier. ${ }^{13}$ A simulated model of the single-crystal Fe(salen) structure and the crystal unit cell are shown in Figure 1b, i and ii, respectively. The $\mathrm{Fe}($ salen) crystal structure was examined using a low-dose electron-beam HRTEM technique (Figure 1c, i) in order to minimize electron-irradiation damage. ${ }^{27,28}$ Figure $1 \mathrm{c}$, ii shows a typical HRTEM image with lattice spacings of 1.06 and $0.99 \mathrm{~nm}$, which correspond to the FFT pattern (Figure 1c, iii), providing evidence for interplanar distances between salen-salen planes and face-to-face stacking, respectively. HRTEM images of other spots were also thoroughly examined (Supplementary Figure S1a, Supplementary Information) and did not indicate the presence of any significant impurities or artifacts. To further confirm the purity of Fe(salen), elemental mapping under scanning TEM (STEM) and energy dispersive X-ray spectroscopy were performed, and the elemental intensities of $\mathrm{Fe}, \mathrm{N}$ and $\mathrm{O}$ were observed (Supplementary Figure S1b). No other elements were detected. Fe(salen) possesses a singlecrystalline organo-structure, distinct from inorganic iron oxides, with a spacing of $\sim 0.25 \mathrm{~nm},{ }^{29}$ and the corresponding SAED gave projected dimensions of $0.85 \times 0.70 \mathrm{~nm}$ with an angle of $92.5^{\circ}$ (Supplementary Figure S2), suggesting that the crystallographic orientations can be obtained in a minimally invasive imaging fashion, which is possibly suitable for further examination of the metal complex configuration. ${ }^{28}$ All these results confirm a 'magnetite-free', high-quality, organic single-crystal nanostructure.

\section{Preparation of Fe(salen)-loaded core-shell nanoassemblies}

$\mathrm{Fe}$ (salen) was loaded into the PCL domain of PP by hydrophobic interactions, forming a CSNA structure (Scheme 1, iv). The drugloading efficiency was determined stoichiometrically to be $\sim 45 \mathrm{wt} \%$, with a DLC of approximately $90 \mathrm{wt} \%$, which is consistent with the $15.4 \%$ atomic-mass value of $\mathrm{Fe}$ (salen) in the CSNAs determined by ICP-mass spectrometry (Supplementary Figure S3b), in contrast to the spectrum of Fe(salen) alone (Supplementary Figure S3a). In conventional methods using MNPs, DLC is typically only $\sim 50 \%$ due to the large intrinsic size of the MNPs $(\sim 50 \mathrm{~nm}) .{ }^{5}$ For example, DLCs of up to $53 \mathrm{wt} \%$ were obtained using cisplatin. ${ }^{30}$ The high DLC that we obtained can be ascribed to the strong non-covalent interaction of PCL-Fe(salen) and the high PCL fraction along with the large surface area of the PCL matrix and the substantially lighter weight of $\mathrm{Fe}$ (salen) compared to that of iron-oxide NPs. This is favorable for pharmaceutical applications, as the total dose of the formulation can be minimized. Extraordinarily, we discovered that the CSNAs are rapidly attracted to a permanent magnet; however, there is no interaction for the $\mathrm{Fe}$ (salen) methanol solution. This enabled rapid separation using a magnet, which is a unique feature of the present methodology. Moreover, we confirmed that a mixture of $\mathrm{FeCl}_{3}$ with PP showed no magnetism and did not undergo chemical reaction (Supplementary Figure S4). Thus, our method is simple, mild, environmentally friendly and inexpensive, in contrast to conventional methods, which require the use of harsh conditions and toxic solvents/ reagents. To our knowledge, this is the first 'green' protocol for the simple synthesis of magneto-nanocarriers without any requirement for the addition of separate MNPs.

\section{Structural characterization of CSNA}

TEM showed that the Fe(salen) $(1 \times)$-loaded CSNAs are amorphous granular nanostructures roughly $50 \mathrm{~nm}$ in size (Figure 2a, i, ii). These structures are aggregated, presumably due to their hydrophobic nature (low doping level (25\%) by $p$-toluenesulfonate). To locate Fe(salen) inside the micellar matrix, FFT analyses were carried out by focus modulation from $300 \mathrm{~nm}$ to $800 \mathrm{~nm}$ with in-focused (Supplementary Figure S5a) or under-focused modulation (Supplementary Figures S5b and $2 \mathrm{~b}$ ), in order to obtain enhanced phase contrast with a uniform transmission density. Unfortunately, direct identification of $\mathrm{Fe}$ (salen) inside the CSNA in defocused HRTEM images (Figure 2b, Supplementary Figure S5b) proved challenging due to the small molecular size of $\mathrm{Fe}($ salen; $\sim 1 \mathrm{~nm}$ ) as well as the interference from the surrounding cloudy PP-matrix. STEM-energy dispersive X-ray spectroscopy analysis (Figure 2c) showed a somewhat higher intensity of elements derived from the $\mathrm{Fe}($ salen) CSNA compared with the results for $\mathrm{Fe}$ (salen) itself (Supplementary Figure S1b). Very few ( 1\%) NCs encapsulated by PPy shells were detected (Supplementary Figure S5c and d), while a higher density of NCs $(\sim 10 \%)$ in the $\mathrm{Fe}($ salen; $1.5 \times)$ CSNA were observed, as shown in Supplementary Figure S6a, i. These showed clear lattice fringes of polycrystalline Fe oxide (Supplementary Figure S6a, ii). The FFT pattern showed distinct rings (Supplementary Figure S6a, iii), confirming the NC-containing CSNA structures. Well-defined NCs with clustered domains were found in TEM analysis and electron energy loss spectroscopy analysis (Supplementary Figure S6b), which are consistent with the XRD analysis (Figure 2d). The CSNA samples showed a broad amorphous peak at $20-30^{\circ}$; it was difficult to observe sharper diffractions from the $\mathrm{Fe}$ (salen) cores inside the CSNA matrix in the XRD pattern, presumably due to the broad diffractions from the amorphous polymeric shells of the CSNAs. These findings 
a

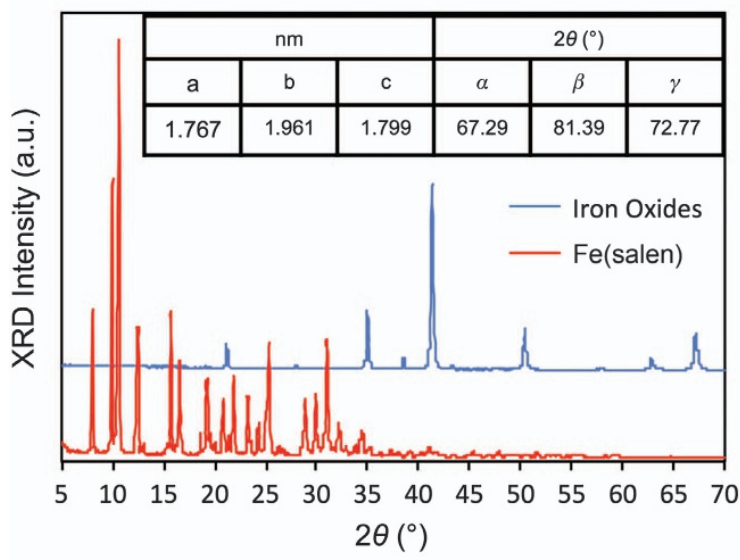

b

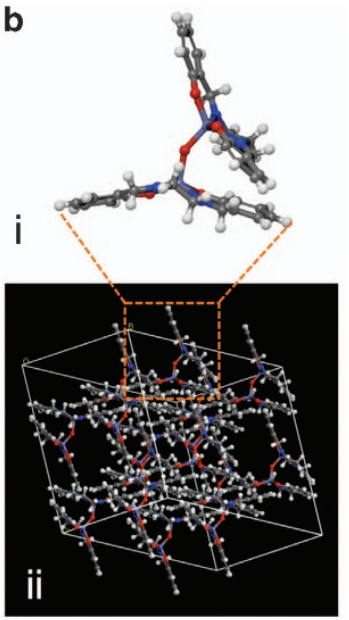

C
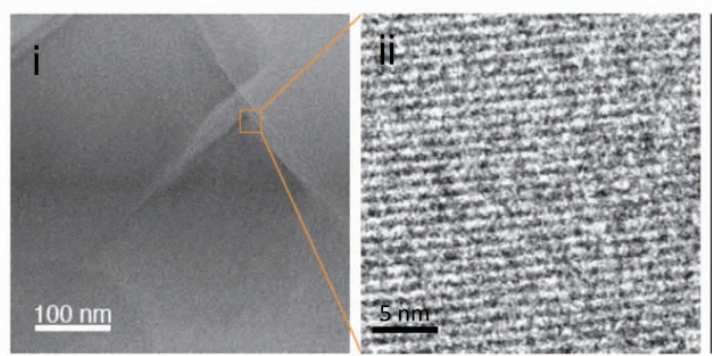

iii

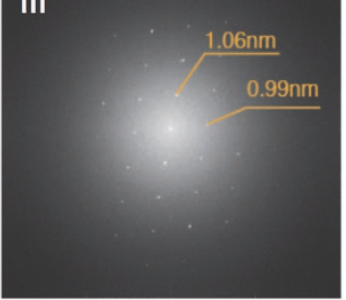

Figure 1 Structural analyses of the purified Fe(salen). (a) X-ray diffraction (XRD) crystallographic analysis accompanied with a standard curve for magnetite. The table of associated lattice parameters of Fe(salen) is shown in the inset. (b) The simulated three-dimensional molecular structure and unit cell of the crystal model are presented (white, purple, red, blue and orange atoms represent hydrogen, carbon, oxygen, nitrogen and iron, respectively). (c) Low-dose beam high-resolution transmission electron microscope (HRTEM) images of recrystallized Fe(salen) samples (i, ii) and the corresponding fast Fourier transform (FFT) pattern analyses (iii).

agree with the results of TEM. The CSNAs with $1.5 \mathrm{x}$ Fe(salen) (d, i) showed typical magnetite peaks at $34.5^{\circ}, 41.4^{\circ}, 49.8^{\circ}$, and $67^{\circ}$, while those with $1 \mathrm{x} \mathrm{Fe}($ salen) (d, ii) showed only negligible peaks at these positions, suggesting that magnetite may be formed by the condensation of the $\mathrm{Fe}$ (salen) molecules within the PP-boundary in samples with high levels of Fe(salen). This result may indicate that chemical-bond-forming reactions, such as oxidation or decomposition, do not occur. Accordingly, CSNAs with $1 \times \mathrm{Fe}$ (salen) were used for further studies. Next, small-angle X-ray scattering measurements of $\mathrm{Fe}$ (salen) and the CSNA samples were performed (Figure 2e) followed by the fitting analysis (Supplementary Figure S7). Assuming a nanosphere boundary, both curves showed nearly identical size distribution ranges from $\sim 1 \mathrm{~nm}$ (high intensity; Figure 2f) to $25-66 \mathrm{~nm}$ (low intensity; Figure $2 \mathrm{f}$, inset). The $\sim 1-\mathrm{nm}$ region corresponds to single $\mathrm{Fe}($ salen) molecules inside the CSNAs, while the minor peaks at $25-66 \mathrm{~nm}$ correspond to grains or NCs, in accordance with the TEM analyses (Supplementary Figure S5c and d).

\section{Biomolecule-induced core-modulation of CSNA mediated by the PPy shell}

To functionalize the CSNA surface to improve water solubility and colloidal stability, we selected BSA and GA, which are biocompatible. The 75\% undoped surface of PPy in the outer shell is hydrophobic, which could allow non-covalent interactions to occur with the hydrophobic entities of BSA and GA molecules. TEM observation of the BSA-coated CSNA (Figure 3a) showed mainly $\sim 2 \mathrm{~nm}$ NCs with single crystallinity inside the amorphous PPy-shell matrixes and a fairly uniform interspacing, which was revealed by FFT analysis
(Figure 3a, ii) as \#1 and \#2. The energy dispersive X-ray spectroscopy results showed a noticeable peak for Fe (Supplementary Figure S8a). As BSA molecules are chemically neutral and should not interact with $\mathrm{Fe}($ salen), it is extraordinary that $\mathrm{Fe}($ salen) spontaneously formed clusters inside the PCL polymer matrix, as such clustering was not seen in charged dispersants. In the case of GA, almost all the Fe(salen) cores grew to larger $(\sim 40 \mathrm{~nm}) \mathrm{NCs}$, as revealed in TEM analyses (Figure $3 \mathrm{~b}$ and Supplementary Figure S8b); these appeared analogous to the structure of $\mathrm{NC}$-containing CSNAs with $1.5 \times \mathrm{Fe}$ (salen). In contrast to the BSA-CSNAs, the formation of larger NCs can be attributed to the attachment of anionic (electron-rich) GA. Thus, we believe that the core size of the $\mathrm{Fe}$ (salen) clusters can be tuned by selecting suitable biomolecules (electron-dopants) to interplay with PPy (electron-mediator). Moreover, it is noteworthy that these processes resemble endogenous magnetite formation in magnetotactic bacteria, such as Magnetospirillum magneticum, in which the Mms6 protein regulates the nanocrystal morphology in biomineralization, ${ }^{31}$ excluding the use of an Fe salt as the Fe source.

\section{Optical properties}

In the UV-vis absorbance spectroscopy (Figure 4a), the surface of the nanoassemblies exhibited a typical PPy signature, with loss of the absorbance peaks at 288 and $310 \mathrm{~nm}$ due to the CSNAs' interior components (PCL and Fe(salen)). This confirms that the $\mathrm{Fe}$ (salen) loading inside PPy was successful. The CSNAs also display a near-infrared peak at $700-800 \mathrm{~nm}$ as a characteristic bipolaron state of the sulfonate-doped PPy polymer. ${ }^{32}$ The FT-IR spectra also confirmed the screening of $\mathrm{Fe}$ (salen) by PPy in the CSNA, compared 
a

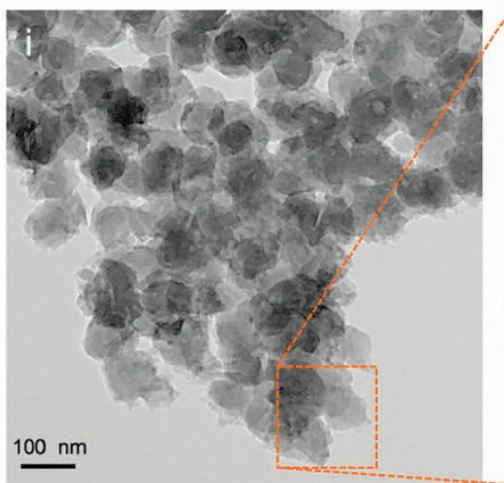

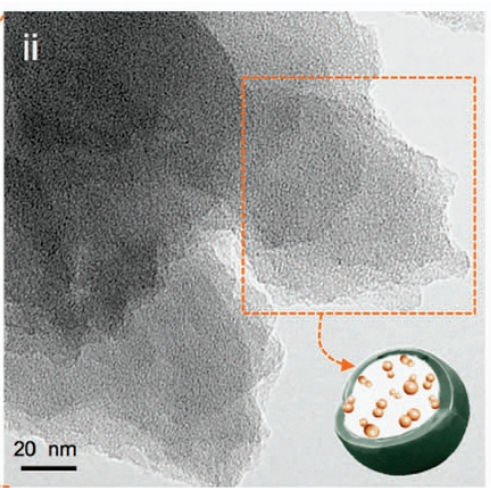
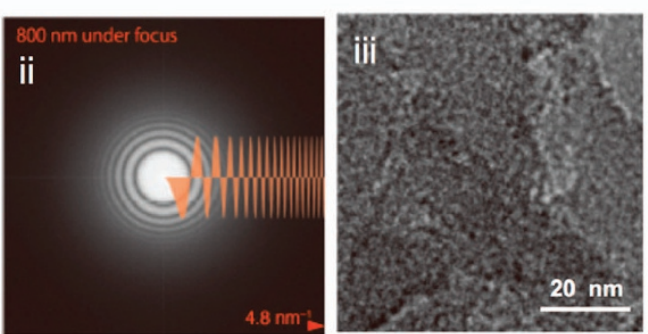

C

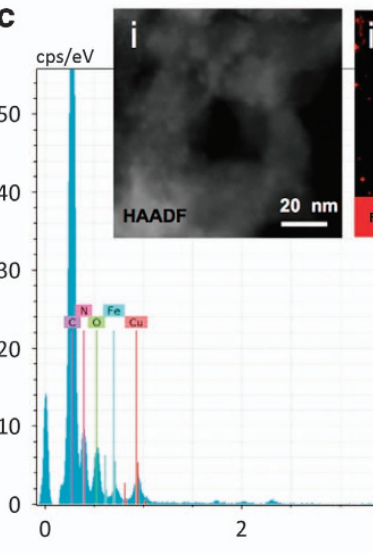

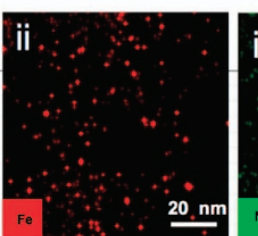
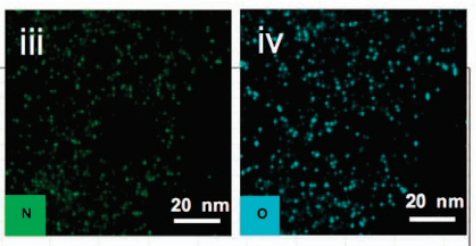

d

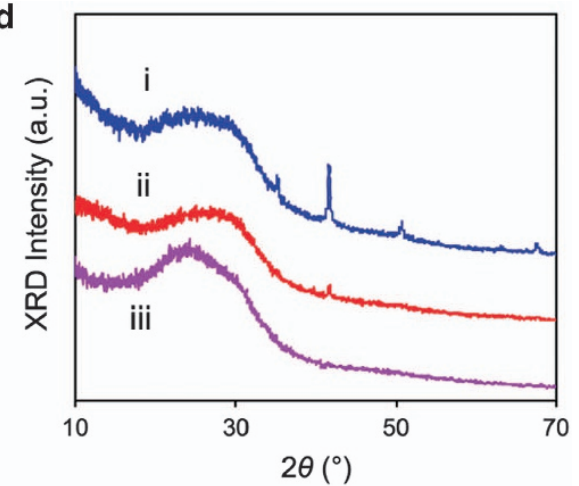

e

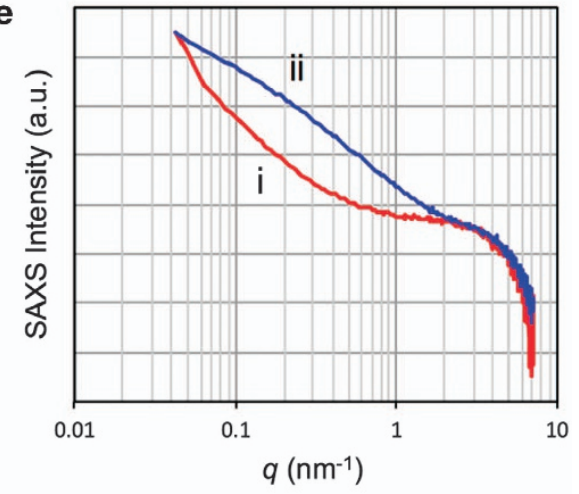

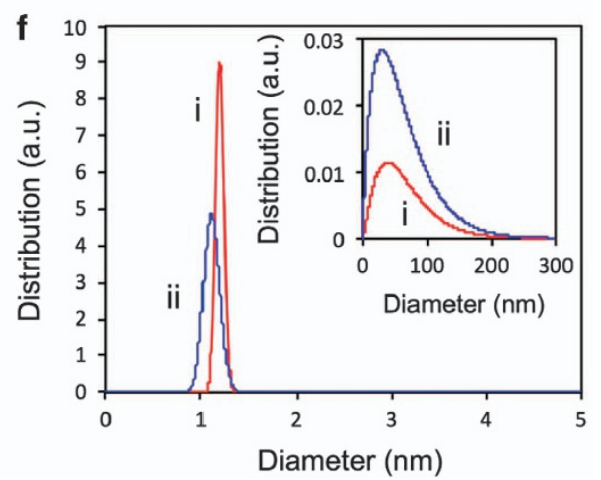

Figure 2 Physico-chemical characterizations of the Fe(salen)-loaded CSNA. (a) Typical transmission electron microscope (TEM) images and (b) a low-dose TEM analysis of the CSNA. (i) The low-resolution TEM image, (ii) the fast Fourier transform (FFT) pattern under-focused at $800 \mathrm{~nm}$ (the superimposed phasecontrast transfer function was used to estimate the magnitude of the under-focus), (iii) high-resolution transmission electron microscope (HRTEM) image of the corresponding TEM-FFT analyses of the square area in (i). (c) The scanning TEM (STEM)-energy dispersive X-ray spectroscopy analysis and mapping, showing slightly increased intensity of Fe that may imply that Fe(salen) molecules are located closer together (clustered) inside the matrix. (d) X-ray diffraction (XRD) analyses of the CSNAs with various concentrations of Fe(salen) (1.5× (i), $1 \times$ (ii) and $0 \times$ (iii)), showing the peaks of the endogenously produced nanoclusters (NCs) inside the CSNA matrix. (e) Small-angle X-ray scattering (SAXS) analysis of Fe(salen) (i) and the CSNAs (ii). Scattering was observed in two samples with a scattering vector of $0.1<q\left(\mathrm{~nm}^{-1}\right)<10$. (f) The mathematically fitted lognormal size distributions in the SAXS analysis.

with free Fe(salen; Figure 4b). The FT-IR spectrum of the purified $\mathrm{Fe}$ (salen) shows typical IR bands of Fe(salen) at 1619, 1384, 1336 and $1302 \mathrm{~cm}^{-1}$, while no vibration bands of $\mathrm{Fe}-\mathrm{O}$ at approximately $580 \mathrm{~cm}^{-1}$ (related to magnetite) were seen. In addition, a band due to asymmetric $\mathrm{Fe}-\mathrm{O}-\mathrm{Fe}$ stretching with a $\mathrm{Fe}-\mathrm{O}-\mathrm{Fe}$ angle of $110-120^{\circ}$ appeared at $700-750 \mathrm{~cm}^{-1}{ }^{33}$ The FT-IR spectrum of Fe(salen) CSNA shows characteristic bipolaron bands at 910 and $1200 \mathrm{~cm}^{-1}$ (refs 32,34) due to S-O and C-S stretching modes of the PPy shell doped with $p$-toluenesulfonate in the range of $500-700 \mathrm{~cm}^{-1} .32,34 \mathrm{We}$ observed $\mathrm{C}=\mathrm{C}$ stretching vibrations at $1562,1434 \mathrm{~cm}^{-1}, \mathrm{C}-\mathrm{N}$ and $\mathrm{C}-\mathrm{C}$ vibrations at $1359 \mathrm{~cm}^{-1}, \mathrm{C}-\mathrm{H}$ in-plane bending vibrations at 1198 and $1142 \mathrm{~cm}^{-1}$, and $\mathrm{C}-\mathrm{H}$ out-of-plane bending vibrations at $780-1033 \mathrm{~cm}^{-1} .32,34$ The peak at $3400 \mathrm{~cm}^{-1}$ is assigned to an $\mathrm{N}-\mathrm{H}$ stretching vibration. These peaks seem to be due to the PPy main chains since they remained almost unmodified in the course of CSNA formation. The UV-vis (Figure 4c) and FT-IR spectra (Figure 4d) also showed characteristics of BSA and GA on the CSNA surface, confirming successful bio-functionalization of the CSNAs. Interestingly, the GA-coated CSNAs showed no absorbance peak at $700-800 \mathrm{~nm}$ due to the formation of large NCs. The colloidal properties of the CSNAs were evaluated using dynamic light scattering and zeta potential measurements (Figure $4 \mathrm{e}$ and f, respectively). Compared with the size obtained in TEM analysis, larger CSNAs $(\sim 300 \mathrm{~nm})$ were observed, which implies that the colloidal CSNAs 
a
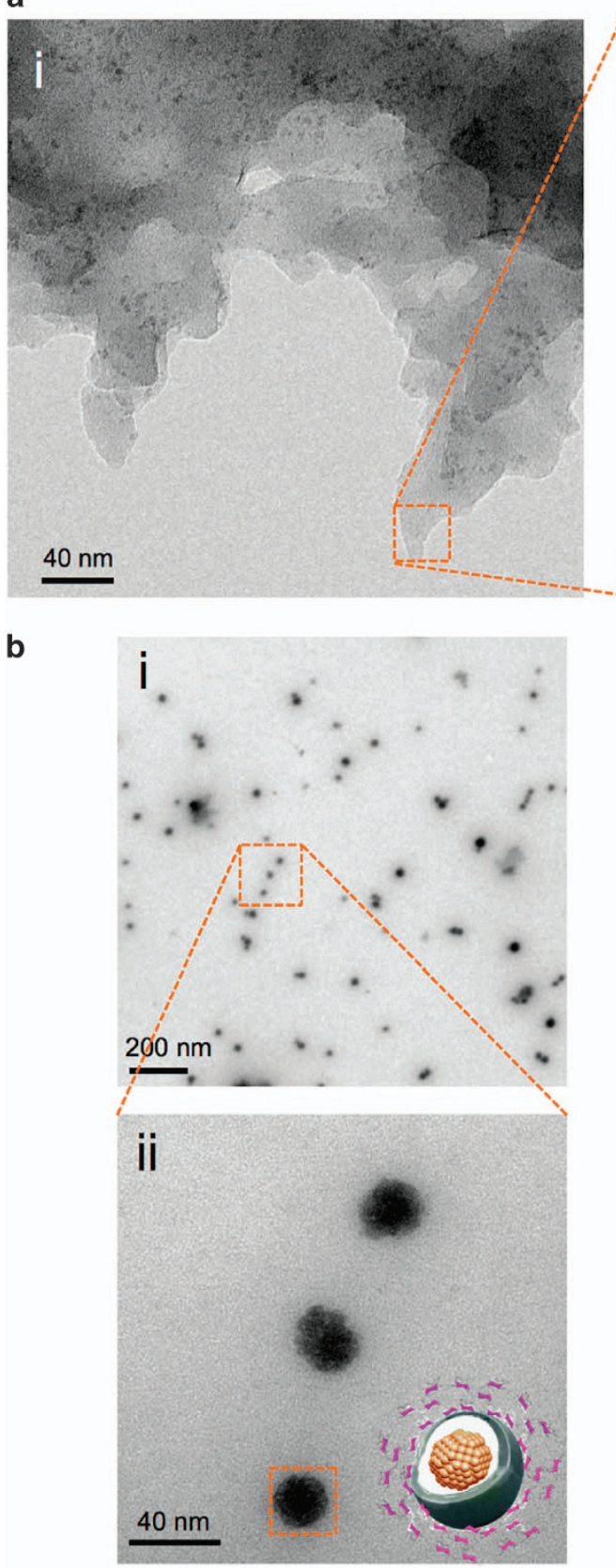
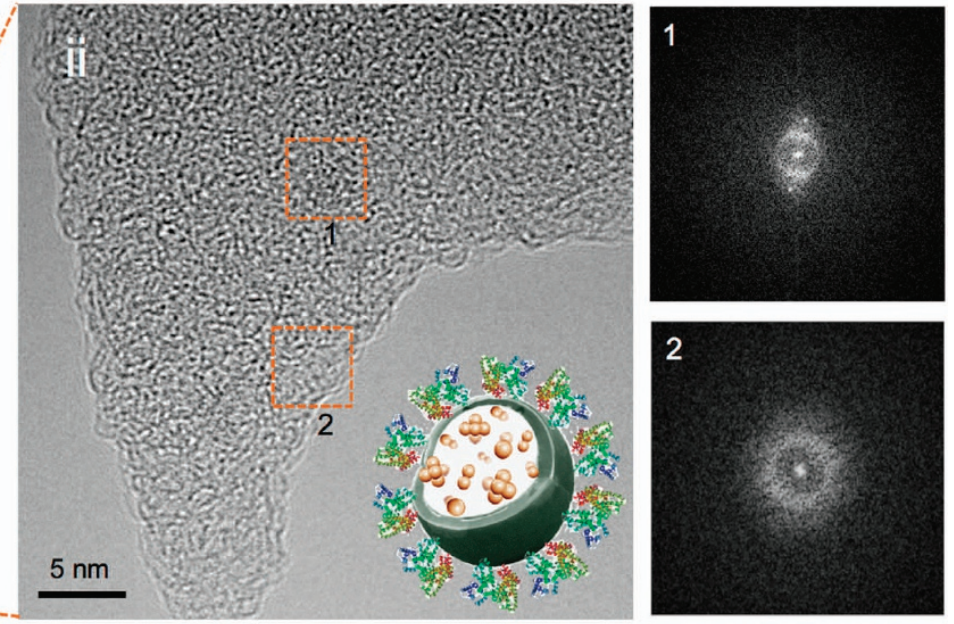

b
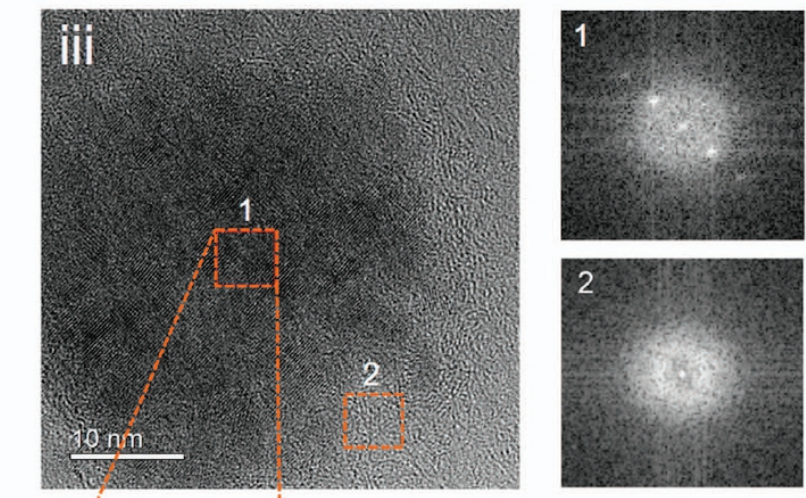

iv

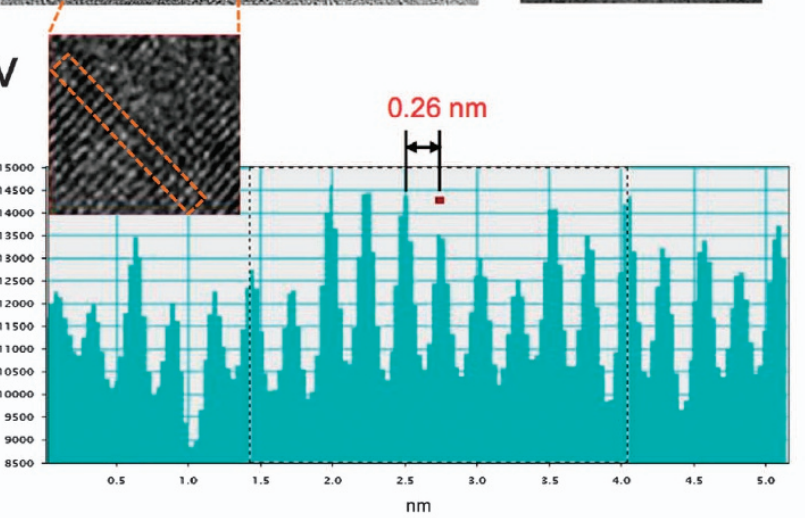

Figure 3 Core-size modulation of the Fe(salen) CSNA by bio-functionalization. (a) Transmission electron microscope (TEM) image of the bovine serum albumin (BSA)-induced CSNA showing small nanoclusters that were endogenously produced inside the CSNA matrix. (i) A low-magnification TEM image of the bovine serum albumin (BSA)-CSNA, (ii) high-resolution transmission electron microscope (HRTEM) image and the corresponding fast Fourier transform (FFT), showing the single nanocrystalline structure of the NC (\#1) and the amorphous structure of the polypyrrole (PPy) matrix region (\#2), and (b) TEM image of the GA-CSNA showing enlarged nanoparticles. (i, ii) Monodispersed CSNA, (iii) a magnified TEM image of a marked one in (ii), showing typical FFT of an iron-oxide polycrystalline structure (\#1) and the amorphous structure of a polypyrrole (PPy) matrix region (\#2) and (iv) the well-defined crystalline lattice fringes with $0.26 \mathrm{~nm}$ interspacing from the marked square \#1.

were swollen in the solution phase. The values of the polydispersity index for BSA-CSNAs, GA-CSNAs and bare CSNAs were $0.272 \pm 0.007,0.339 \pm 0.032$ and $0.352 \pm 0.021$, respectively, confirming high monodispersivity. The zeta potential values were $-25,-30$ and $+26 \mathrm{mV}$, respectively, representing successful surface bio-passivation and high colloidal stability. The CSNAs remained stable for at least 6 months, based on the dynamic light scattering and zeta potential analyses (Supplementary Figure S9).

\section{Magnetic properties}

Precise magnetic analysis of iron compounds is often challenging, since even minute amounts of impurities, such as oxide or carbide, can alter the magnetism, resulting in inaccurate measurements. Hence, to examine the magnetic properties of $\mathrm{Fe}$ (salen), the thoroughly purified $\mathrm{Fe}$ (salen) was completely dissolved in methanol, where it was evenly distributed in the solution. The liquid sample showed no apparent magnetic response in ESR analysis due to magnetite 

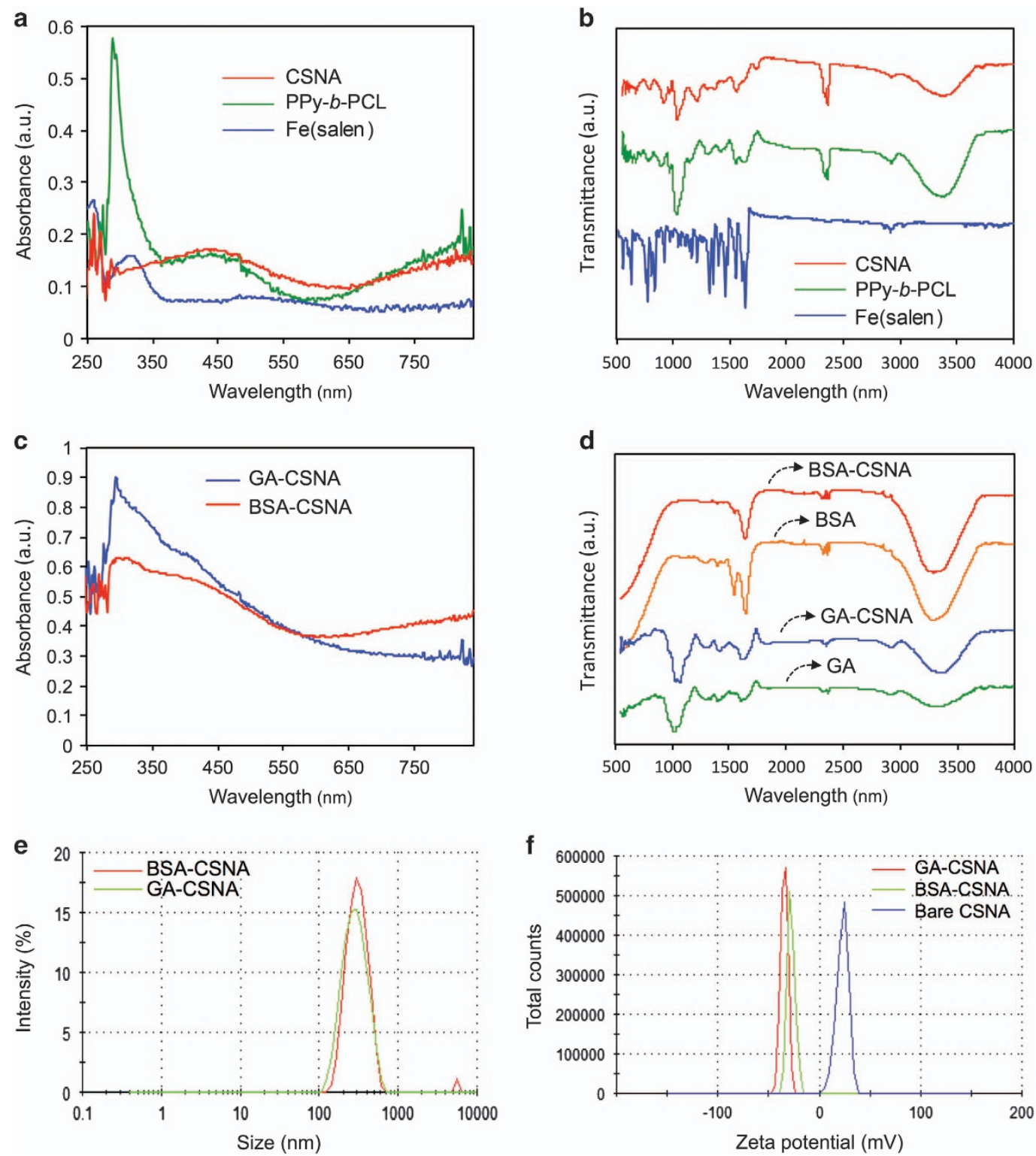

Figure 4 Optical characterizations of the Fe(salen)-loaded CSNAs. (a) Ultraviolet-visible (UV-vis) spectra and (b) fourier transform infrared (FT-IR) spectra of the Fe(salen)-loaded CSN compared with free $\mathrm{Fe}($ salen) and the polypyrrole (PPy)-b-polycaprolactone (PCL) polymer. Characterization of the bio-functionalization by the (c) UV-vis spectra and (d) FT-IR spectra, confirming surface coating of bovine serum albumin (BSA) or gum arabic (GA). (e) dynamic light scattering size distribution and (f) zeta potential analysis of the CSNA samples.

impurities (Supplementary Figure S10). To further characterize the magnetic properties of the purified $\mathrm{Fe}$ (salen) and the CSNAs, we performed temperature-dependent magnetization measurements at $2-300 \mathrm{~K}$, as shown in Supplementary Figure S11 and Figure 5a-d, respectively. Noticeably, magnetization curves of the CSNAs at $300 \mathrm{~K}$ (Figure 5b) exhibited distinct hysteresis with a coercivity of $60 \mathrm{Oe}$, equal to that of $\mathrm{Fe}$ (salen). Moreover, the relatively low $H \mathrm{c}$ value and its temperature dependence $(2-300 \mathrm{~K})$ indicate that magnetized CSNAs exhibit magnetic softness, suggesting that the spins of the CSNAs form a homogeneous arrangement. The magnetization intensity of the purified $\mathrm{Fe}$ (salen) at $2 \mathrm{~K}$ was decreased at $50 \mathrm{~K}$ and stabilized up to $300 \mathrm{~K}$ (a), as similarly discussed in our previous report, ${ }^{13}$ while the coercivity was constantly decreased as the temperature increased. This feature can also be seen in the curves of the CSNAs at $2 \mathrm{~K}(\mathrm{a}, \mathrm{b})$ and $300 \mathrm{~K}(\mathrm{c}, \mathrm{d})$. Remarkably, the magnetization of the CSNAs is $\sim 5$ times larger than that of $\mathrm{Fe}($ salen) itself. The ESR results (Figure 5e) and the magnetic properties by attaching a magnet (Figure 5e, inset) were similar in all cases. We also performed temperature-dependent magnetization of ZFC and field cooling at 2-300 K (Figure 5f). At a low magnetic field (100 Oe), all the CSNAs, including Fe(salen) samples, exhibited a spin-glass-like behavior ${ }^{35}$ with a high blocking temperature ( $200 \mathrm{~K}$; Figure $5 \mathrm{f}$ ), while no blocking temperature was observed at 1-10 kOe (Supplementary Figure S12). Particularly, at a low temperature range of 2-25 K, the magnetization in ZFC steeply decreased due to the antiferromagnetic-like phase transition, assuming that the antiferromagnetic coupling dominates the ferromagnetic (FM) coupling, which is a typical feature of a spin-glass state that has competition between FM and antiferromagnetic interaction. ${ }^{35}$

To assign the oxidation state and spin state of $\mathrm{Fe}($ salen), we performed ${ }^{57} \mathrm{Fe}$ Mössbauer spectroscopy measurements from 4.2 to 
a

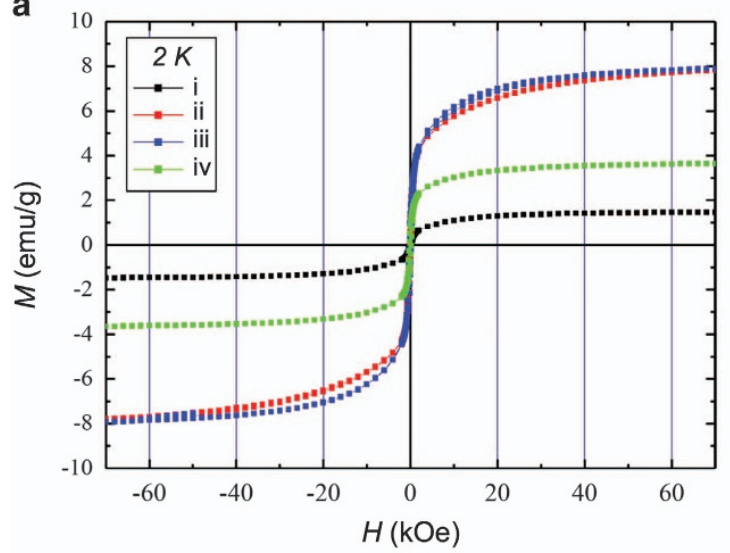

C
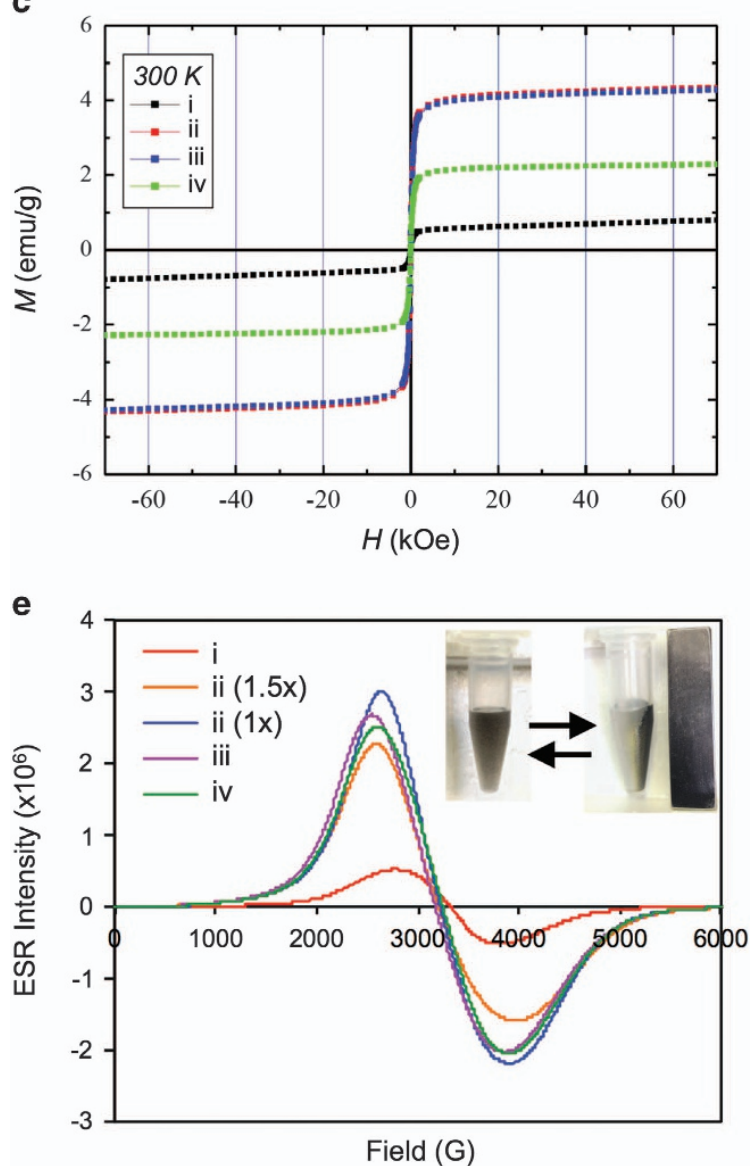

b

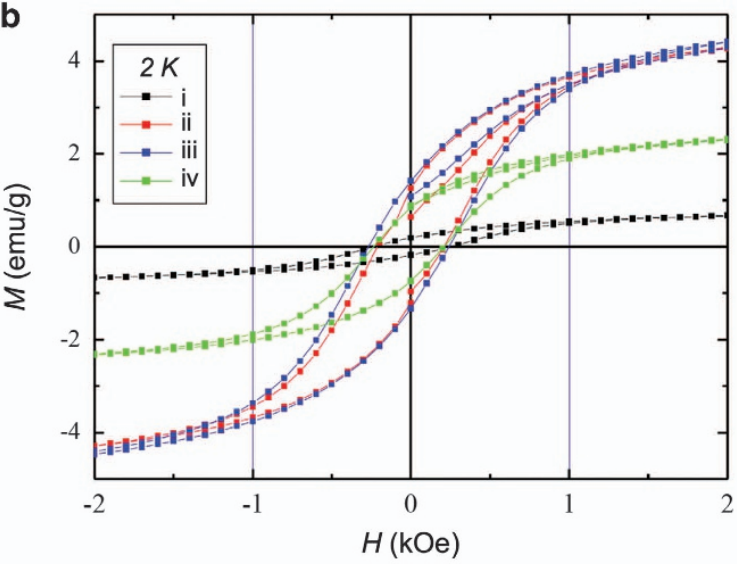

d

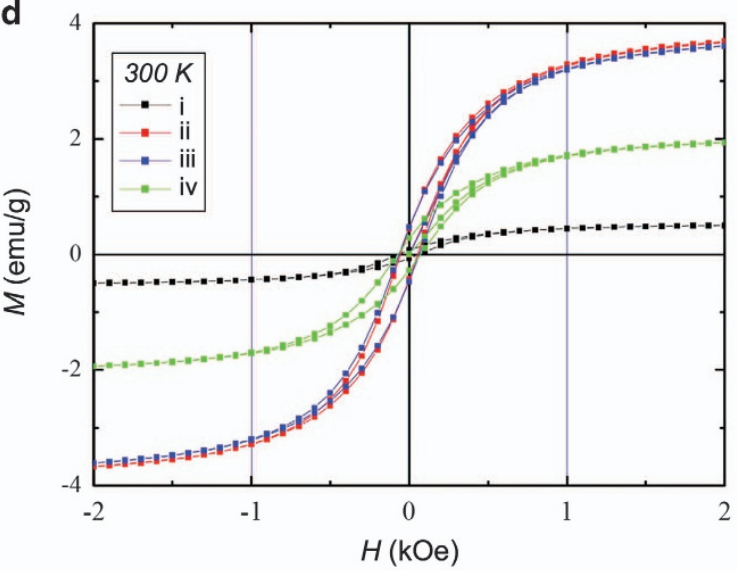

$\mathbf{f}$

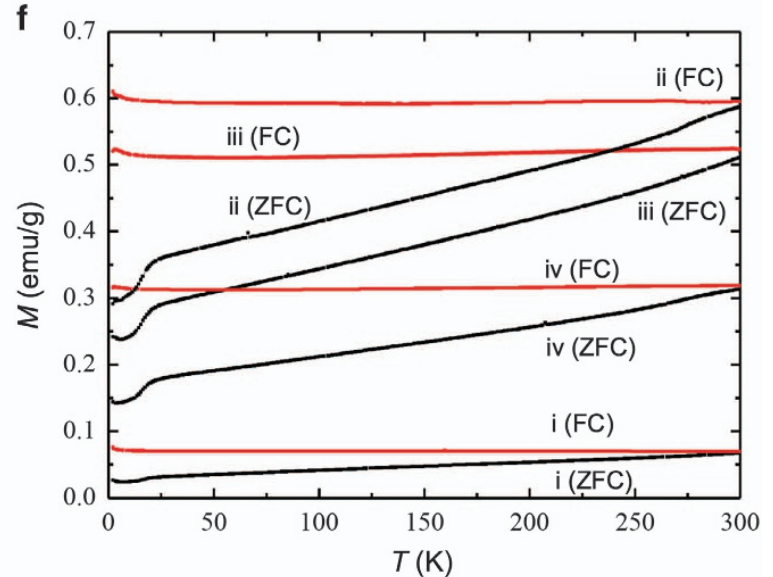

Figure 5 Magnetization analyses of Fe(salen), the Fe(salen)-loaded CSNAs and the bio-functionalized Fe(salen)-loaded CSNAs. The comparative temperaturedependent magnetic hysteresis curves of Fe(salen) (i), Fe(salen)-loaded CSNAs (ii), bovine serum albumin (BSA)-coated CSNAs (iii) and gum arabic (GA)-coated CSNAs (iv) were plotted from $2 \mathrm{~K}$ ( $\mathbf{a}$ and $\mathbf{b}$ ) to $300 \mathrm{~K}$ (c and $\mathbf{d}$ ). The low-field portion on the right-hand side (b and $\mathbf{d}$ ) shows the temperature-dependent HC value. (e) Electron spin resonance (ESR) curves of all the CSNAs, including $1.5 \times$ Fe(salen)-loaded CSNAs. The inset shows a photo of magnet-guided attraction of the CSNA dispersion in saline solution (all CSNAs are visually identical). (f) Low magnetic field-dependent zero field cooling (ZFC)-field cooling (FC) magnetic properties at 100 Oe.

298.15 K. The ${ }^{57} \mathrm{Fe}$ Mössbauer spectrum of the Fe(salen) complex exhibited a dominant doublet with a quadrupole splitting at $0.45 \mathrm{~mm} \mathrm{~s}^{-1}$ and the isomer shifts of $0.3535(298.15 \mathrm{~K})$, $0.4579(77.4 \mathrm{~K})$ and $0.4691(4.2 \mathrm{~K}) \mathrm{mm} \mathrm{s}^{-1}$, which lies in the range of typical high-spin $(S=2)$ coordinated Fe(II) complex species. ${ }^{36}$ However, the valence state of $\mathrm{Fe}($ salen) was not accurately determined in this method. As investigated in a similar study ${ }^{37}$ of the oxidation state of $\mathrm{Fe}$ (salen) using ${ }^{57} \mathrm{Fe}$ Mössbauer spectroscopy, Fe(salen) may favorably take either divalent states or trivalent sates. Rather, from first-principles spin-density functional calculations in our previous study, ${ }^{13}$ we found that Fe was present as a high-spin $(S=2)$ divalent ion with substantial covalency involving the ligands, indicating that $\mathrm{Fe}$ (salen) is a hypoligated complex. ${ }^{38}$ This finding was consistent with our previous experimental analysis using cyclic voltammetry. ${ }^{13}$ 
a

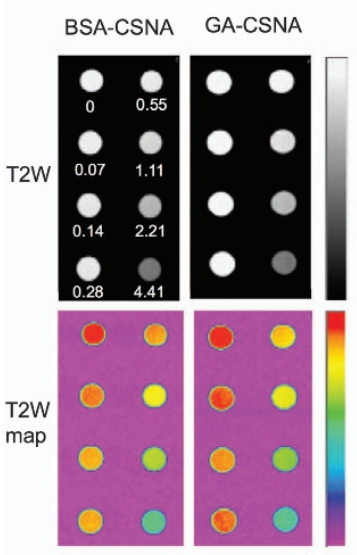

b
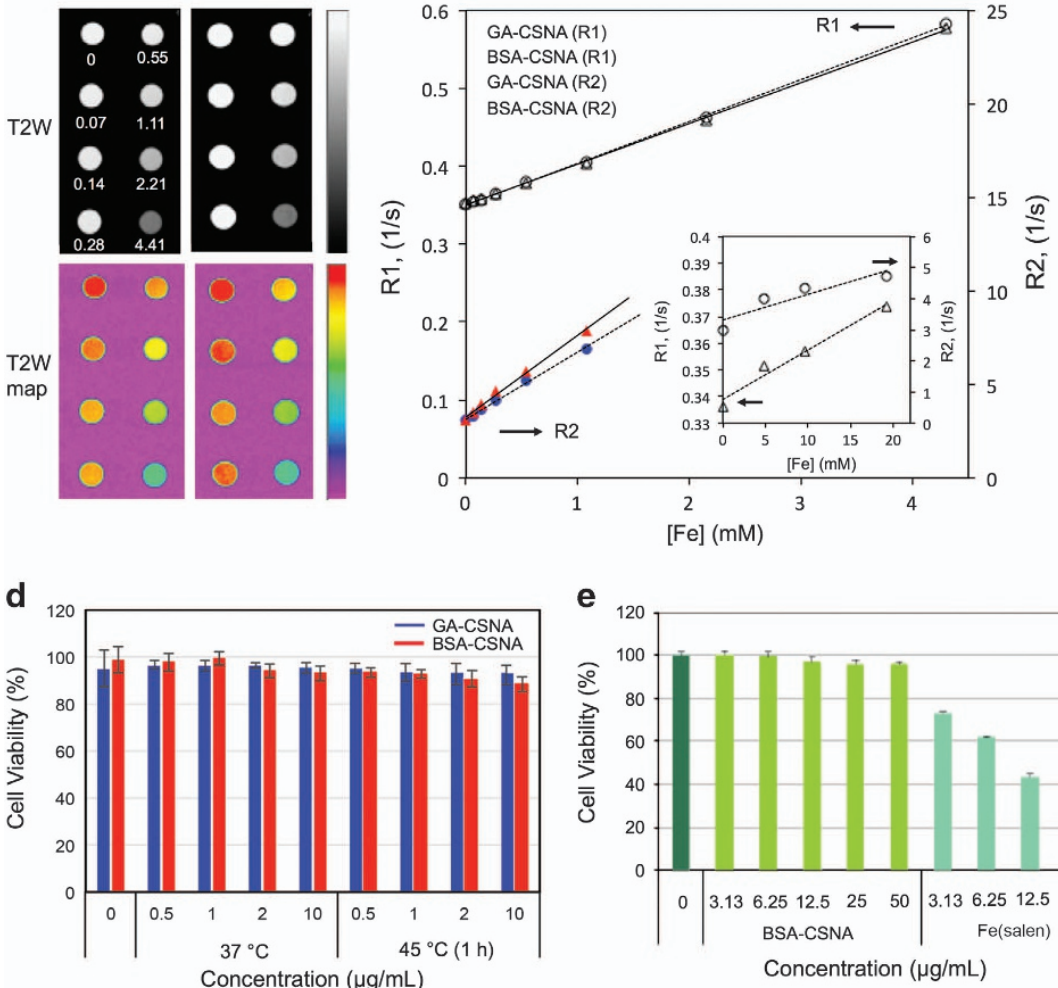

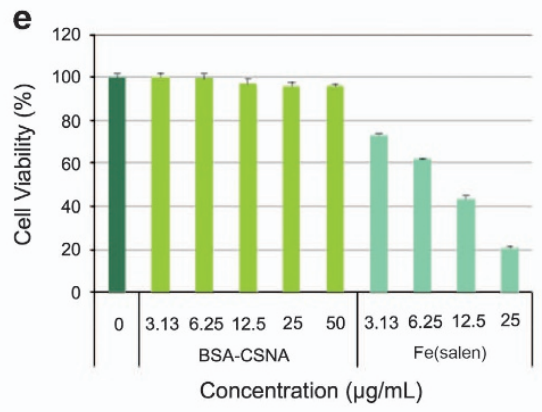

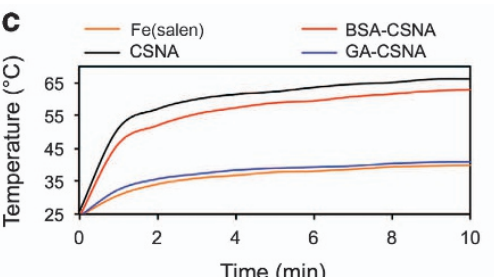
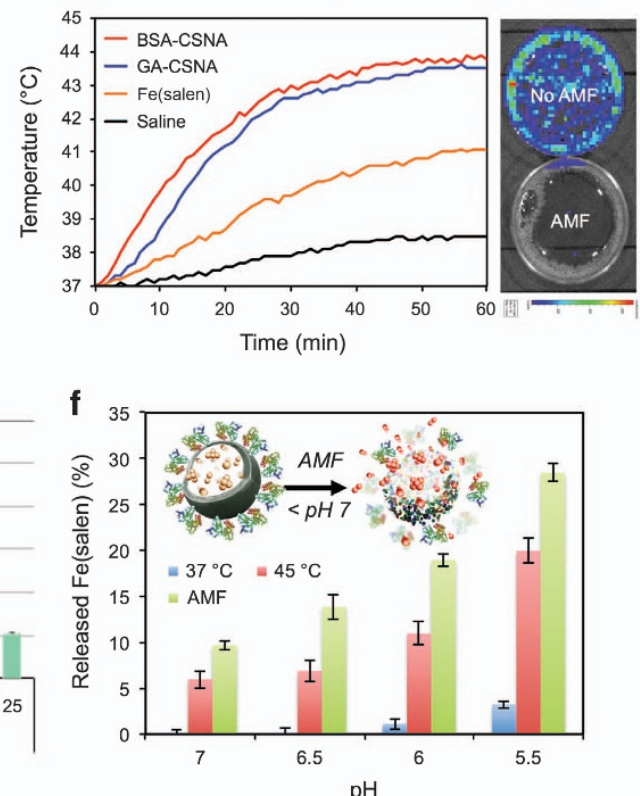

Figure 6 Multifunctional capabilities of the CSNAs (Fe(salen)-loaded CSNAs). (a) Magnetic resonance images of the bovine serum albumin (BSA)- or gum arabic (GA)-coated CSNAs. A sample of each low concentration (0-4.41 mm) was acquired with a 7-Tesla scanner for T2W and mapping. (b) The proton longitudinal relaxation rate (R1) and transverse relaxation rate (R2) of the bio-functionalized CSNA samples were calculated quantitatively to confirm linearity in contrast to Fe(salen). (c) AMF-induced heat generation profiles of the CSNA products in powder (the top) and saline solution (the bottom), and the in vitro fluorescent cytotoxicity assay (in vivo imaging system) using the BSA-CSNAs (the right panel). (d) HS-SY-II cell proliferation assay of the response to Fe (salen) release under external heating, showing outstanding thermo-stability. (e) HS-SY-II cytotoxicity assay of the BSA-CSNAs versus Fe(salen) as a function of concentration. (f) Release profiles of Fe(salen) from a BSA-CSNA suspension in the pH-dependent AMF-heating mode.

To further examine the oxidation state of the Fe ions in $\mathrm{Fe}$ (salen), XPS was employed. The XPS spectrum of $\mathrm{Fe}\left(\right.$ salen) (Fe 2p $\left.p_{3 / 2}\right)$ (red line) was scanned in the range of $700-740 \mathrm{eV}$, as shown in Supplementary Figure S13. The binding energy found for the Fe $2 p_{3 / 2}$ peaks in $\mathrm{Fe}($ salen) was $710.8 \mathrm{eV}$, while the reference binding energy was set to $284.6 \mathrm{eV}$ for $\mathrm{C} 1 \mathrm{~s}$. This value lies within the expected range for chemically stable Fe(II) centers bound to a Schiff-base ligand (salen), for which the peak is typically $710.5-711 \mathrm{eV}$, representing that $\mathrm{Fe}$ (salen) exists in a divalent state, as correlated elsewhere. ${ }^{39}$ When compared with $\mathrm{Fe}$ (salen) $\mathrm{Cl}$ (monomer, blue line), the binding energies of $\mathrm{Fe}$ (salen) at $\sim 710$ and $725 \mathrm{eV}$ were shifted $0.4-1 \mathrm{eV}$ lower than that of $\mathrm{Fe}$ (salen) $\mathrm{Cl}$, supporting that $\mathrm{Fe}($ salen) is in a divalent state, which is in good agreement with a previous study. ${ }^{40}$ However, we could not achieve a defined XPS spectrum of $\mathrm{Fe}($ salen) inside the CSNA matrix due to an interference by the amorphous PPy shell of the CSNAs, as similarly discussed in the section of XRD analysis.

Combining all the given results, including $\mu_{\mathrm{B}}$ values, ${ }^{57} \mathrm{Fe}$ Mössbauer spectroscopy, XPS and theoretical analyses, possible explanation for the size-tunable NC formation and the remarkable magnetic enhancement over standalone $\mathrm{Fe}$ (salen) include the following two models, which are discussed in detail below: the magnetic coupling model and the magnetic domain model. First, we will discuss the magnetic coupling model (Supplementary Figure S14). Correlating the core-shell micellar interfaces to the electronic states of Fe in the
CSNAs, suggests that the increased FM intensity can be attributed to the PPy-doping degree, resulting in an increase of unpaired electrons in the $\mathrm{d}^{5}$ orbital of $\mathrm{Fe}^{2+}$, as discussed elsewhere. ${ }^{41,42}$ For instance, FM coupling can occur between $p$-toluenesulfonate-doped $p$-phenylenediamine radicals in $\pi$-conjugated PPy chains and $\mathrm{Fe}$ (salen)-cores. Consequently, the PPy shells appear to play a key role in regulating electron-transfer into the core, where $\mathrm{Fe}^{2+} / \mathrm{Fe}^{+}$atoms serve as electron-acceptors, resulting in NC formation.

In the bare nanoassembly system, shown in Supplementary Figure S14a (i), the doped PPy-shell interface can offer $n$ electron to reduce $\mathrm{Fe}^{2+}$ in the $\mathrm{Fe}\left(\right.$ salen) center, converting $\mathrm{Fe}$ into an $\mathrm{Fe}^{+}$state, which may contribute to an increased FM state. With an added electron, the spin in each orbital of Fe is favored to be delocalized via the double-exchange mechanism, which leads to an FM coupling along with the increased spin state $(S>2)^{43}$ (Supplementary Figure S14b). In the BSA-coated CSNA system (ii), the formation of uniform clusters can be attributed to the attachment of the BSA, an electron-donor, on the surface of the doped PPy shell, where it is assumed to amplify electron-transfer from BSA to the Fe(salen)containing interior. The Fe centers, as electron-acceptors, can then be partially reduced from $\mathrm{Fe}^{+}$to $\mathrm{Fe}^{0}$, favoring cluster formation but coexisting with $\mathrm{Fe}^{+}$(salen). In contrast to the small NCs in the BSA-CSNA, the larger NCs can be attributed to the attachment of GA, an electron-rich polyelectrolyte, to the PPy shell. As GA has 
a larger electron density than BSA, most of the Fe centers can be reduced to form single-core $\mathrm{Fe}^{0}$ (salen) CSNAs. The fewer electrons of $p$-toluenesulfonate or BSA would offer higher FM coupling than the excess electrons of GA. Now, we will discuss the magnetic domain model. Compared with the bare- or BSA-CSNAs, large NC-containing CSNAs (that is, GA-CSNAs and $1.5 \times \mathrm{Fe}$ (salen)-loaded CSNAs) showed reduced magnetism, which seems surprising given that FM intensity is usually proportional to NP granular size. ${ }^{44}$ Thus, we consider that an $\sim 50-\mathrm{nm}$ particle in the GA-CSNAs contains randomly disordered domains, whereas that in the BSA-CSNAs contains relatively homogenous single domains, retaining high ferromagnetism. According to the Weiss theory, the magnetization of the latter should be far higher, ${ }^{45}$ offering modulatable magnetism of the MNP. All these results are consistent with the idea that the magnetism of $\mathrm{Fe}($ salen) is intrinsic and not due to contaminating factors. As the electronic properties of the $\mathrm{Fe}($ salen) core changes under different charge transport environments, there is a possibility that the Fe cores inside the CSNAs also possess mixed valence states in the dimer complexes, for example, $\mathrm{Fe}^{2+}-\mathrm{Fe}^{+}$or $\mathrm{Fe}^{+}-\mathrm{Fe}^{0}$. However, the effect of the FM of intramolecular spin transport through such an exchange-coupled mixed-valence system is experimentally challenging to undercover, and thus, this remains unexplored to date. ${ }^{38}$ Therefore, more intensive studies in both experiment and theory are required to determine the critical magnetic factors for high anisotropy of Fe(salen) NCs (for example, spin states, spin orders and spin coupling) inside the CSNAs. More intensive studies are also needed to investigate surface effects on coercivity, blocking temperature and so on.

\section{MRI and relaxometry}

We have discovered that bio-functionalized Fe(salen)-loaded CSNAs can be visualized by MRI due to the Fe-concentration-dependent $(0-4.4 \mathrm{~mm})$ negative signal change in T2W and mapping (Figure 6a). We observed an increase of both the linear longitudinal relaxation rate $\left(\mathrm{R} 1\left(\mathrm{~s}^{-1}\right)\right)$ and the transverse relaxation rate $\left(\mathrm{R} 2\left(\mathrm{~s}^{-1}\right)\right)$, and evaluation of the longitudinal relaxivity $\left(r_{1}\right)$ and transverse relaxivity $\left(r_{2}\right)$ gave the following values (Figure 6b): M-BSA $r_{1}=0.05$ $\left(\mathrm{mM}^{-1} \mathrm{~s}^{-1}\right), \quad r_{2}=4.36 \quad\left(\mathrm{mM}^{-1} \mathrm{~s}^{-1}\right)$ and M-GA $r_{1}=0.05$ $\left.\left(\mathrm{mM}^{-1} \mathrm{~s}^{-1}\right), r_{2}=3.59\left(\mathrm{mM}^{-1} \mathrm{~s}^{-1}\right)\right)$ over the concentration ranges of $0-4.4 \mathrm{~mm}$ Fe for $r_{1}$ and $0-1.1 \mathrm{~mm}$ Fe for $r_{2}$ (in the linear regions $\left.\left(R^{2}>0.99\right)\right)$. These values represent substantially increased values with higher linearity compared with $\mathrm{Fe}($ salen), as shown in Figure 6b (inset; $r_{1}=0.002\left(\mathrm{mM}^{-1} \mathrm{~s}^{-1} ; R^{2}=0.97\right), r_{2}=0.0824$ $\left.\left(\mathrm{mM}^{-1} \mathrm{~s}^{-1} ; R^{2}=0.84\right)\right)$. Thus, MRI can be used to estimate the local concentration, aiding dose optimization of $\mathrm{Fe}$ (salen) in a clinical setting with a low-dose injection. Usually, larger Fe NPs have stronger saturated magnetization (Ms) and higher transverse relaxivity $\left(r_{2}\right){ }^{46}$ Conversely, comparing with the GA-coated CSNAs with larger cores, the BSA-CSNAs with multiple smaller NCs showed slightly improved intensity on T2W contrast in the low concentration range $(0-1.1 \mathrm{mM})$ as well as slightly higher $r_{2}$ relaxivity, in good agreement with the as-described magnetic properties.

\section{Magneto-hyperthermia}

Irradiation with an AMF caused the temperature of powdered CSNA, BSA-CSNA and GA-CSNA samples at $25^{\circ} \mathrm{C}$ to increase to $51^{\circ} \mathrm{C}$, $46.3^{\circ} \mathrm{C}$ and $32.4^{\circ} \mathrm{C}$, respectively, within $1 \mathrm{~min}$, showing dramatically greater heat production than the $\mathrm{Fe}\left(\right.$ salen) powder sample $\left(30.7^{\circ} \mathrm{C}\right)$. The exceptional enhancement of magneto-thermal sensitivity of the CSNA samples is also in accordance with the as-discussed magnetic properties (superconducting quantum interference device, ESR and MRI) given from the magnetic coupling of the conductive polymer shell of the CSNAs. In a physiological environment (saline at $37^{\circ} \mathrm{C}$ ), the BSA- or GA-CSNA suspension was heated to $43.0^{\circ} \mathrm{C}$ or $42.6^{\circ} \mathrm{C}$, respectively, within $30 \mathrm{~min}$, showing substantially greater heat generation than $\mathrm{Fe}$ (salen) in saline $\left(39.7^{\circ} \mathrm{C}\right)$, while saline alone showed a small increase $\left(37.9^{\circ} \mathrm{C}\right)$ (Figure 6c). The effective magnetic heating was acquired by the diluted low sample concentration $\left(0.625 \mathrm{mg} \mathrm{ml}^{-1}\right)$ of the BSA-CSNAs, which is far below the conventional level in iron-oxide NP-based in vitro studies $\left(>5 \mathrm{mg} \mathrm{ml}^{-1}\right)$. Bare CSNA samples were unable to be used for further in vitro studies due to their poor water solubility, while the GA-CSNAs showed less hyperthermal effect than the BSA-CSNAs. AMF-induced in vitro hyperthermia for $1 \mathrm{~h}$ caused $>99 \%$ cell death in in vivo imaging system assays (Figure 6c, inset), confirming the remarkable magnetohyperthermal efficacy of the BSA-CSNAs. As a superior advantage of magnetic DDS, this would allow the use of a relatively small dose of injected sample, since it can be accumulated on a desired local spot by magnetic attraction, enabling enhanced AMF-exposure and heat generation in contrast with the in vitro batch, where the particles were diluted and dispersed. We have recently explored the in vivo magnetic guided hyperthermia method, ${ }^{14}$ showing effective AMF-heating by injecting a concentration of only $\sim 30 \mu \mathrm{M}$, which makes us believe that the given BSA-CSNA samples could enable improved hyperthermic performance in future pre-clinical stages.

\section{Controlled drug release}

The bio-functionalized CSNAs showed good stability under external heating $(\mathrm{EH})$ at $45^{\circ} \mathrm{C}$ for $1 \mathrm{~h}$, releasing only $\sim 6 \%$ of the $\mathrm{Fe}$ (salen) in the case of the BSA-CSNAs and only $\sim 2 \%$ in the case of the GA-CSNAs (Figure 6d). Correspondingly, both CSNAs did not exhibit significant cytotoxicity at $37^{\circ} \mathrm{C}$. However, after activation by $\mathrm{EH}$ at $45^{\circ} \mathrm{C}$ for $1 \mathrm{~h}, \sim 10 \%$ of the cells were dead in the BSA-CSNA-containing sample at $10 \mu \mathrm{g} \mathrm{ml}^{-1}$, compared with relatively low cell death $(\sim 6 \%)$ in the GA-CSNAs. In a concentrationdependent cytotoxicity test (Figure 6e), the BSA-CSNAs did not show noticeable cytotoxicity up to $50 \mu \mathrm{g} \mathrm{ml}^{-1}$ due to the well-stabilized surface functionalization, while $\mathrm{Fe}$ (salen) significantly killed the cells at $25 \mu \mathrm{g} \mathrm{ml}^{-1}$. Unless AMF-treatment was engaged, the BSA-CSNAs showed similar biosafety and robust stability against other cancer cell lines including ovary cancer (OVK18) and glioblastoma (U87; Supplementary Figure S15), which predicts clinical security for minimizing side effects toward various cells. Compared with the $\mathrm{EH}$-induced low release rate of $\mathrm{Fe}$ (salen) from the BSA-CSNAs (5-6\%) or GA-CSNAs ( 2\%) (Supplementary Figure S16a), lowering the solution $\mathrm{pH}$ from 7 to 4.5 at $37^{\circ} \mathrm{C}$ for $1 \mathrm{~h}$ caused release of $\sim 8 \%$ of the $\mathrm{Fe}$ (salen) from the BSA-CSNAs and $\sim 1.5 \%$ from the GA-CSNAs (Supplementary Figure S16b). Thus, the CSNAs may comprise two stimulus-responsive sections: heat-sensitive PCL (melting at $>42{ }^{\circ} \mathrm{C}$ ) and $\mathrm{pH}$-sensitive PPy (swelling at $<\mathrm{pH} 7$ from a CSNA suspension in the $\mathrm{pH}$-dependent AMF-heating mode). This implies that simultaneous heating and $\mathrm{pH}$ change could maximize drug release. Indeed, $\mathrm{Fe}($ salen) release from the BSA-CSNAs by AMF-induced heating under acidic $\mathrm{pH}$ conditions (Figure 6f) increased the drug release to $14 \%$ at $\mathrm{pH} 6.5$ and $19.5 \%$ at $\mathrm{pH} 6.0$, compared with the EH-induced acidic platform $(7 \%$ at $\mathrm{pH} 6.5 ; 11 \%$ at $\mathrm{pH} 6.0$ ). This result supports the idea that the PPy shell plays a key role in the $\mathrm{pH}$-responsive release. This also suggests that the BSA-CSNAs securely preserve the anticancer activity of $\mathrm{Fe}$ (salen) by employing a large group of small clusters and enabling the release through the nanopores of PPy when the PCL is melted under heating (the anticancer activity is a direct proof of existence of intact Fe(salen) inside the CSNAs). The NCs in the 

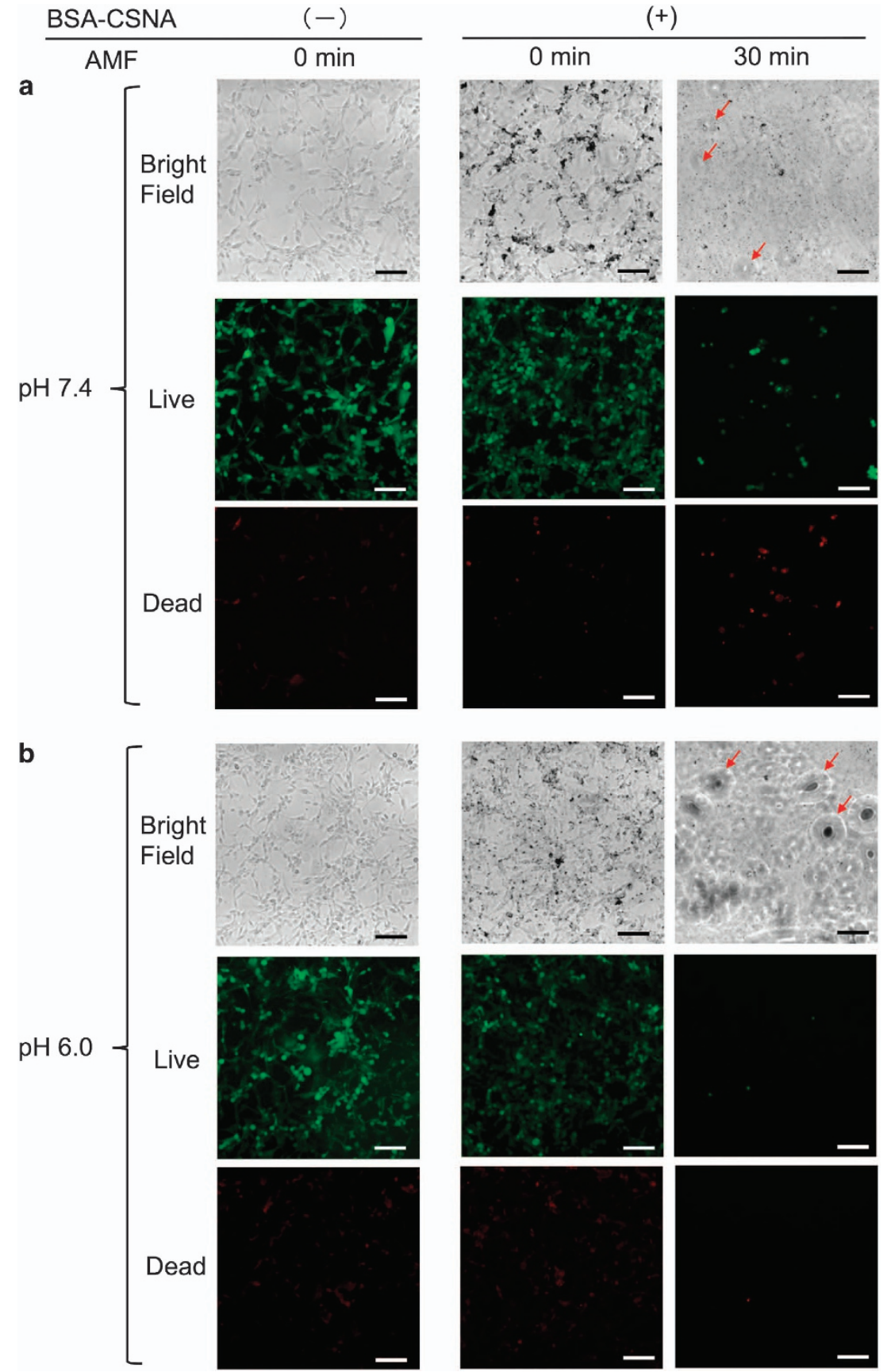

Figure 7 Microscopic imaging of human glioblastoma (U87) cells to evaluate the magneto-hyperthermal drug release efficacy of the bovine serum albumin (BSA)-CSNAs in response to dual stimuli (alternating magnetic field (AMF) and $\mathrm{pH}$ ). The AMF-hyperthermal anticancer treatment was performed at $\mathrm{pH} 7.0$ (a) and $\mathrm{pH} 6.0$ (b). When exposed to AMF for $30 \mathrm{~min}$, the cells at pH 6.0 were mostly dead, while they were partially viable at pH 7.4 . A significant number of floating (dead) cells is shown in the bright field images (red arrows) after AMF-treatment for 30 min, which is not detectable in the fluorescence imaging. Scale bars $=200 \mu \mathrm{m}$.

GA-CSNAs turned out to be inert rather than cytotoxic, due to a loss of the intact drug activity led by intensified NC formation.

To validate the rationale behind the $\mathrm{pH}$ - and hyperthermia-induced enhancement of the anticancer activity of the BSA-CSNA, we performed in vitro magnetic hyperthermia treatments at different physiological $\mathrm{pH}$ values. In this investigation, we employed U87 cells to evaluate the potent cytotoxicity of the BSA-CSNA formulation against brain cancer. The morphology of the control and of the BSA-CSNA treated cells were observed by microscopic imaging
(Figure 7 and Supplementary Figure S17a), which showed that the cells were viable in the presence of the BSA-CSNAs. Particularly, black-colored entities of the BSA-CSNAs were shown on the cells, implying the uptake or adhesion of the BSA-CSNAs by the cells, without showing any loss of viability (the slightly darkened green fluorescence is assumed to be due to a photoquenching of the organic dye molecules in the presence of the PPy shells of the CSNAs). Both bright field and fluorescence cell imaging demonstrated a significant increase in AMF-dependent toxicity of the BSA-CSNAs. 
Most of the cells at both $\mathrm{pH}$ values were dead after AMF-irradiation for $60 \mathrm{~min}(\sim 85.9 \%$ at $\mathrm{pH} 7.4 ; \sim 91.4 \%$ at $\mathrm{pH} 6.0)$, which did not allow us to clarify the effect of $\mathrm{pH}$ (Supplementary Figure S17a). On the other hand, under reduced irradiation for $30 \mathrm{~min}$, only $\sim 8.1 \%$ of the cells survived at $\mathrm{pH} 6.0$, while $\sim 37.4 \%$ of the cells were still viable at $\mathrm{pH} 7.4$ (Supplementary Figure S17b). This reveals that the $\mathrm{Fe}($ salen) molecules loaded in the BSA-CSNAs were released by the AMF-irradiation under the acidic conditions, as evidenced by a comparison with the AMF-treated sample at $\mathrm{pH} 7.4$ as well as the sample without AMF-treatment at $\mathrm{pH}$ 6.0. Taking into account the release profile of the BSA-CSNAs at $\mathrm{pH} 6.0(\sim 20 \%)$ as demonstrated in Figure 6f, the released amount of $\mathrm{Fe}($ salen) was assumed to be $\sim 281.25 \mu \mathrm{g}$ equivalent $\mathrm{Fe}($ salen$)$ per $\mathrm{ml}$, which falls far past the dose range beyond $\sim 80 \%$ cell death efficiency of $\sim 40 \mu \mathrm{g} \mathrm{ml}^{-1}$ $\mathrm{Fe}$ (salen). ${ }^{13,14}$ It is noteworthy that the combination of a drug, magnetic hyperthermia and $\mathrm{pH}$ can lead to more effective cell targeting in comparison to the individual treatments of the hyperthermia or drug. In addition, it is not only promising for low-doseenabled minimally invasive hyperthermal therapy with shortened therapeutic exposure (AMF) time but also favorable for effective drug release because the majority of extracellular tumor environments are slightly acidic. Moreover, the biocompatible and stable nature of the BSA-CSNA colloids would protect the drug payload from undesirable events, that is, enzymatic degradation and protein bio-fouling, which often occur in in vivo systems, for example, blood and serum. These characteristics could be utilized to design a low-dosage system in acidic conditions for water-insoluble drug payloads. For instance, the $\mathrm{Fe}$ (salen)-loaded CSNAs might show favorable release characteristics for intracellular cancer targeting in acidic tumor lysosomes $(\mathrm{pH} \sim 5)$, as well as in proliferating macrophages in atherosclerosis lesions ( $\mathrm{pH}$ 5.5), with minimal side effects on normal cells.

\section{CONCLUSION}

In summary, instead of using iron-oxide NPs as an exclusive mainstay for conventional magnetic DDS platforms, we have developed a simple and convenient method for preparing micelle-like CSNA composites loaded with $\mathrm{Fe}$ (salen), which has both intrinsic antitumor activity and magnetic properties. These single-drug CSNAs have a large loading capacity and exhibit stable colloidal properties because they contain lightweight drug molecules rather than inorganic metal NPs. In addition, they are readily core-and surface-modifiable with BSA or GA, giving them extraordinary physico-chemical versatility: they are highly soluble in water, easy to process, biocompatible and magnetically tunable. The as-prepared CSNAs are multifunctional; they can be imaged by means of MRI, and their intrinsic magnetism enables AMF-induced hyperthermia. In particular, the BSA-coated CSNAs exhibited AMF- and pH-triggered $\mathrm{Fe}$ (salen) release in addition to the hyperthermal effect, enabling not only 'on-demand' drug delivery while inhibiting undesirable cytotoxic effects but also synergistic 'chemo-thermal-therapy' in a single anticancer system. With these outstanding features, the BSA-CSNAs appear to be a potential integrated nanohybrid system combining magnetic hyperthermia with drug delivery, offering many advantages over existing inorganic MNP systems and suitability for pre-clinical applications. In addition, in future work, a similar synthetic strategy may also be applicable to fabricate magnetized nanocarriers for the co-loading of insoluble anticancer drugs, such as paclitaxel, methotrexate or doxorubicin. Optimizing the surface-coating design, self-assembly conditions and magnetic properties should open up a new paradigm for the development of prodrug-based versatile magnetic DDS nanocarriers for the effective theranostic treatment of cancer.

\section{CONFLICT OF INTEREST}

The authors declare no conflict of interest.

\section{ACKNOWLEDGEMENTS}

We are grateful to A. Nagasako, C. Oyamada, M. Katsumata and H. Aoyama for technical assistance in this study. We also thank N. Nitta, Y. Ozawa and S. Sayaka (National Institute of Radiological Sciences, QST) for MRI measurements. Special thanks to K. Yoshida (Japan Fine Ceramics Center) and T. Watanabe (FEI Gatan Inc. Japan) for the TEM tomography. This work was supported in part by the Japan Society for the Promotion of Science (IS), a Grant-in-Aid for JSPS Fellows (IS). This study was also supported in part by the Japan Society for the Promotion of Science (JSPS) KAKENHI Grant (25670131 to YI); The Ministry of Education, Culture, Sports, Science and Technology (MEXT) KAKENHI Grant (22136009 to YI); New Energy and Industrial Technology Development Organization (NEDO; 60890021 to YI); the National Cerebral and Cardiovascular Center (NCVC; 22-2-3 to YI); the Japan Agency for Medical Research and Development (AMED; 66890005, 66890011, 66890001 and 66890023 to YI); and The Tokyo Biochemical Research Foundation (YI). The MRI system and quantification technology are partly supported by a COI grant (MEXT, Japan).

Author contributions: JHK, HE and IY proposed the research direction and guided the project. JHK designed and performed the syntheses of the samples and the physico-chemical and optical analyses. JHK, MU and IS conducted the biological studies. HE conducted the simulation studies. SY and TM carried out superconducting quantum interference device magnetization analyses. JHK and YH performed chemical analyses. KS and MY performed physico-chemical analyses. All authors analyzed and discussed the experimental results. JHK drafted the manuscript.

1 Lee, N., Yoo, D., Ling, D., Cho, M. H., Hyeon, T. \& Cheon, J. Iron oxide based nanoparticles for multimodal imaging and magnetoresponsive therapy. Chem. Rev. 115, 10637-10689 (2015)

2 Sao, R., Vaish, R. \& Sinha, N. Multifunctional drug delivery systems using inorganic nanomaterials: a review. J. Nanosci. Nanotechnol. 15, 1960-1972 (2015).

3 Carregal-Romero, S., Guardia, P., Yu, X., Hartmann, R., Pellegrino, T. \& Parak, W. J. Magnetically triggered release of molecular cargo from iron oxide nanoparticle loaded microcapsules. Nanoscale 7, 570-576 (2015).

4 Baek, S., Singh, R. K., Khanal, D., Patel, K. D., Lee, E.-J., Leong, K. W., Chrzanowski, W. \& Kim, H.-W. Smart multifunctional drug delivery towards anticancer therapy harmonized in mesoporous nanoparticles. Nanoscale 7, 14191-14216 (2015).

5 Mou, X., Ali, Z., Li, S. \& He, N. Applications of magnetic nanoparticles in targeted drug delivery system. J. Nanosci. Nanotechnol. 15, 54-62 (2015).

6 Luo, C., Sun, J., Sun, B. \& He, Z. Prodrug-based nanoparticulate drug delivery strategies for cancer therapy. Trends Pharmacol. Sci. 35, 556-566 (2014).

7 Hadjichristidis, N., Hirao, A., Tezuka, Y. \& Prez, F. D. Complex Macromolecular ArcHitectures: Synthesis, Characterization, And Self-Assembly pp. 256 (Wiley, 2011).

8 Gheybi, H. \& Adeli, M. Supramolecular anticancer drug delivery systems based on linear-dendritic copolymers. Polym. Chem. 6, 2580-2615 (2015).

9 Kataoka, K., Harada, A. \& Nagasaki, Y. Block copolymer micelles for drug delivery: design, characterization and biological significance. Adv. Drug Deliv. Rev. 47, 113-131 (2001).

10 Naqvi, S., Samim, M., Abdin, M. Z., Ahmed, F. J., Maitra, A. N., Prashant, C. K. \& Dinda, A. K. Concentration-dependent toxicity of iron oxide nanoparticles mediated by increased oxidative stress. Int. J. Nanomedicine 5, 983-989 (2010).

11 Soenen, S. J., De Cuyper, M., De Smedt, S. C. \& Braeckmans, K. Investigating the toxic effects of iron oxide nanoparticles. Methods Enzymol. 509, 195-224 (2012).

12 Wang, J., Chen, Y., Chen, B., Ding, J., Xia, G., Gao, C., Cheng, J., Jin, N., Zhou, Y., Li, X., Tang, M. \& Wang, X. M. Pharmacokinetic parameters and tissue distribution of magnetic Fe304 nanoparticles in mice. Int. J. Nanomedicine 5, 861-866 (2010).

13 Eguchi, H., Umemura, M., Kurotani, R., Fukumura, H., Sato, I., Kim, J. H., Hoshino, Y., Lee, J., Amemiya, N., Sato, M., Hirata, K., Singh, D. J., Masuda, T., Yamamoto, M., Urano, T., Yoshida, K., Tanigaki, K., Yamamoto, M., Sato, M., Inoue, S., Aoki, I. \& Ishikawa, Y. A magnetic anti-cancer compound for magnet-guided delivery and magnetic resonance imaging. Sci. Rep. 5, 9194 (2015).

14 Sato, I., Umemura, M., Mitsudo, K., Fukumura, H., Kim, J. H., Hoshino, Y. Nakashima, H., Kioi, M., Nakakaji, R., Sato, M., Fujita, T., Yokoyama, U., Okumura, S., Oshiro, H., Eguchi, H., Tohnai, I. \& Ishikawa, Y. Simultaneous hyperthermiachemotherapy with controlled drug delivery using single-drug nanoparticles". Sci. Rep. 6, 24629 (2016).

15 Cong, Y., Wang, Z., He, S., Zhou, D., Li, J., Xie, Z., Chen, X., Jinga, X. \& Huang, Y. Multifunctional single-drug loaded nanoparticles for enhanced cancer treatment with low toxicity in vivo. RSC. Adv. 6, 20366-20373 (2016) 
16 Renfrew, A. K. Transition metal complexes with bioactive ligands: mechanisms for selective ligand release and applications for drug delivery. Metallomics 6, 1324-1335 (2014).

17 Venkataramanan, N. S., Kuppuraj, G. \& Rajagopal, S. Metal-salen complexes as efficient catalysts for the oxygenation of heteroatom containing organic compounds — synthetic and mechanistic aspects. Coord. Chem. Rev. 249, 1249-1268 (2005).

18 Cozzi, P. G. Metal-salen Schiff base complexes in catalysis: practical aspects. Chem. Soc. Rev. 10, 410-421 (2004).

19 Ford, P. C. \& Wecksler, S. Photochemical reactions leading to NO and NOx generation. Coord. Chem. Rev. 249, 1382-1395 (2005).

20 Murray, K. S. Binuclear oxo-bridged iron(III) complexes. Coord. Chem. Rev. 12, 1-35 (1974).

21 Herchel, R., Sindelár, Z., Trávnícek, Z., Zboril, R. \& Vanco, J. Novel 1D chain $\mathrm{Fe}(\mathrm{III})$-salen-like complexes involving anionic heterocyclic $\mathrm{N}$-donor ligands. Synthesis, X-ray structure, magnetic, $57 \mathrm{Fe}$ Mössbauer, and biological activity studies. Dalton Trans. 9870-9880 (2009).

22 Jancsó, A., Paksi, Z., Mikkola, S., Rockenbauer, A. \& Gajda, T. Iron(III)- and copper(II) complexes of an asymmetric, pentadentate salen-like ligand bearing a pendant carboxylate group. J Inorg. Biochem. 99, 1480-1489 (2005).

23 Gatteschi, D., Fittipaldi, M., Sangregorio, C. \& Sorace, L. Exploring the no-man's land between molecular nanomagnets and magnetic nanoparticles. Angew. Chem. Int. Ed. 51, 4792-4800 (2012)

$24 \mathrm{Hao}$, H., Zheng, X., Jia, T. \& Zeng, Z. Room temperature memory device using single molecule magnets. RSC Adv. 5, 54667-54671 (2015).

25 Gatteschi, D., Sessoli, R. \& Villain, J. Molecular Nanomagnets, (Oxford University Press, 2006).

26 Ferlay, S., Mallah, T., Ouahès, R., Veillet, P. \& Verdaguer, M. A room-temperature organometallic magnet based on Prussian blue. Nature 378, 701-703 (1995).

27 Yoshida, K. \& Isoda, S. Control of crystal structure and orientation of Ni(salen) by epitaxial growth on alkali halide. Chem. Mater. 19, 6174-6179 (2007).

28 Yoshida, K., Biskupek, J., Kurata, H. \& Kaiser, U. Critical conditions for atomic resolution imaging of molecular crystals by aberration-corrected HRTEM. Ultramicroscopy 159, 73-80 (2015).

29 Cornell, R. M. \& Schwertmann, U. The iron oxides: structure, properties, reactions, occurrence and uses 2nd edn, (Wiley, 2003).

30 Kim, J., Yoon, H.-J., Kim, S., Wang, K., Ishii, T., Kim, Y.-R. \& Jang, W.-D. Polymermetal complex micelles for the combination of sustained drug releasing and photodynamic therapy. J. Mater. Chem. 19, 4627-4631 (2009).

31 Tanaka, M., Mazuyama, E., Arakaki, A. \& Matsunaga, T. Mms6 protein regulates crystal morphology during nano-sized magnetite biomineralization in vivo. J. Biol. Chem. 286, 6386-6392 (2011).

32 Wu, J., Zhi, Y., Shan, S., Su, H., Wu, S. \& Jia, Q. Polypyrrole nanofibers supported $\mathrm{Cr}(\mathrm{III})($ salen)Cl catalyst: a novel polymer supported catalyst for alternating copolymerization of cyclohexene oxide with carbon dioxide. Catal. Lett. 145, 1913-1921 (2015)

33 Sanders-Loehr, J., Wheeler, W. D., Shiemke, A. K., Averill, B. A. \& Loehr, T. M. Electronic and Raman spectroscopic properties of oxo-bridged dinuclear iron centers in proteins and model compounds. J. Am. Chem. Soc. 111 (21), 8084-8093 (1989).
34 Jang, K.-S., Lee, S. \& Moon, B. Synthesis and characterization of water soluble polypyrrole doped with functional dopants. Synth. Met. 143, 289-294 (2004).

35 Mydosh, J. A. Spin Glasses: An Experimental Introduction 108-110 (Taylor \& Francis, 1993).

36 Scepaniak, J. J., Harris, T. D., Vogel, C. S., Sutter, J., Meyer, K. \& Smith, J. M. Spin crossover in a four-coordinate iron(II) complex. J. Am. Chem. Soc. 133, 3824-3827 (2011).

37 Bancroft, G. M., Maddock, A. G. \& Burns, R. G. Applications of the Mössbauer effect to silicate mineralogy-I. Iron silicates of known crystal structure. Geochim. Cosmochim. Acta 31, 2219 (1967).

38 Pauling, L. The Nature Of The Chemical Bond And The Structure Of Molecules And Crystals: An Introduction To Modern Structural Chemistry (Cornell University Press: Ithaca, 1960).

39 Moulder J. F., Stickle W. F., Sobol P. E. \& Bomben K. D. (eds.) Handbook of X Ray Photoelectron Spectroscopy: A Reference Book of Standard Spectra for Identification and Interpretation of XPS Data, (Perkin-EImer Corporation, 1992).

40 Jain, R., Kabir, K., Gilroy, J. B., Mitchell, K. A. R., Wong, K.-C. \& Hicks, R. G. Hightemperature metal-organic magnets. Nature 445, 291-294 (2007).

41 Shil, S. \& Herrmann, C. Increasing magnetic coupling through oxidation of a ferrocene bridge. Inorg. Chem. 54, 11733-11740 (2015).

42 Yang, H., Jiang, W. \& Lu, Y. Ferromagnetic conductive polypyrrole doped with watersoluble ferrocene derivative. Mater. Lett. 61, 1439-1442 (2007).

43 Soncini, A., Mallah, T. \& Chibotaru, L. F. Molecular spintronics in mixed-valence magnetic dimers: the double-exchange blockade mechanism. J. Am. Chem. Soc. 132 , 8106-8114 (2010).

44 Lee, J. S., Cha, J.-M., Yoon, H.-Y., Lee, J.-K. \& Kim, Y. K. Magnetic multi-granule nanoclusters: a model system that exhibits universal size effect of magnetic coercivity. Sci. Rep. 5, 12135 (2015).

45 Weiss, P. La variation du ferromagnetisme du temperature. Comptes Rendus 143, 1136-1149 (1906) cited in Cullity, 116 (2008).

46 Lee, N., Kim, H., Choi, S. H., Park, M., Kim, D., Kim, H. C., Choi, Y., Lin, S., Kim, B. H., Jung, H. S., Kim, H., Park, K. S., Moon, W. K. \& Hyeon, T. Magnetosomelike ferrimagnetic iron oxide nanocubes for highly sensitive MRI of single cells and transplanted pancreatic islets. Proc. Natl Acad. Sci. USA 108, 2662-2667 (2011).

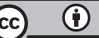

This work is licensed under a Creative Commons Attribution 4.0 International License. The images or other third party material in this article are included in the article's Creative Commons license, unless indicated otherwise in the credit line; if the material is not included under the Creative Commons license, users will need to obtain permission from the license holder to reproduce the material. To view a copy of this license, visit http:// creativecommons.org/licenses/by/4.0/

(C) The Author(s) 2017

Supplementary Information accompanies the paper on the NPG Asia Materials website (http://www.nature.com/am) 